Excited st at es conf $i$ gur at $i$ ons of the quant um Toda I at t i ce

\begin{tabular}{|l|l|}
\hline 著者 & Nat suyana Aki hi ko \\
\hline $\begin{array}{l}\text { j our nal or } \\
\text { publ i cat i on ti t l e }\end{array}$ & Annal s of Physi cs \\
\hline vol une & 289 \\
\hline nunber & 2 \\
\hline page r ange & $178-212$ \\
\hline year & $2001-05-01$ \\
\hline 出版者 & El sevi er \\
\hline 権利 & (c) 2001 Academ c Press. Al I ri ght s reserved. \\
\hline URL & ht t p: //hdl . handl e. net /10297/620 \\
\hline
\end{tabular}




\title{
Excited states configurations of the quantum Toda lattice
}

\author{
A. Matsuyama \\ Department of Physics, Faculty of Science, Shizuoka University, 836 Ohya, Shizuoka \\ 422-8529, Japan
}

Excited states configurations of the quantum Toda lattice are studied by the direct diagonalization of the Hamiltonian. The most probable configurations of one-hole and one-particle excitations are shown to be similar to the profiles of classical phonon and soliton excitations respectively. One-hole excitation states, which are always ground states of definite $E_{m^{-}}$symmetry of the dihedral group $D_{N}$, change those structures abruptly with the potential range varied. One-particle excitations, which are buried in complicated excitation spectra, have well-defined configurations similar to the cnoidal profile of the classical periodic Toda lattice. The relationship that the hole (particle) excitations in quantum mechanics correspond to the phonon (soliton) excitations in classical mechanics, which has been suggested based on the similarity of dispersion relations, is confirmed in a geometrically understandable way. Based on the study of one-soliton and two-soliton states, the structure of multi-soliton states in quantum mechanics can be conjectured.

Key Words: Toda lattice, Soliton, Integrable system 


\section{INTRODUCTION}

Integrable systems have been one of the most growing fields in mathematical physics. They have as many conserved quantities as the degrees of freedom, and thus, their dynamics are severely restricted. Among many integrable systems, onedimensional lattice models such as Heisenberg model and Hubbard model, and continuum models subject to KdV equation and nonlinear Schrödinger equation have been studied in a systematic way. In many cases, they give rise to soliton solutions in classical mechanics and their initial value problems can be treated by using the inverse scattering method. In quantum mechanics, many of them have been solved in terms of Bethe ansatz equations, and found to have a critical point at $T=0$ and gapless excitations. Furthermore their ground state correlation functions are shown to decay asymptotically as powers of the distance.

The important issue is how the properties of the integrable system are related between classical and quantum mechanics. The similarity of the quantum and classical dispersion relations suggests that quantum particle (hole) excitations correspond to classical solitons (phonons $=$ harmonic vibrations) respectively. However, this correspondence is phenomenological and one would like to understand more direct relationships. In other words, where one can find some sort of quantum soliton? Or it may be better to ask whether there exist solitonic objects, i.e., geometrically localized objects, in quantum integrable systems.

The purpose of this paper is to provide new insights into solitonic objects in a typical integrable system, i.e., the quantum Toda lattice. The Toda lattice is one of the most famous integrable models in mathematical physics. In classical mechanics, it has celebrated soliton solutions discovered by Toda [1]. The integrability of the Toda lattice was proved by Hénon [2] and Flaschka [3]. An initial value problem of the infinite open Toda lattice is solved by the inverse scattering method [4], while the solution of the periodic Toda lattice is given by Kac and Moerbeke [5] and Date and Tanaka [6]. The quantum mechanical study of the periodic Toda lattice was first done by Gutzwiller [7] based on the idea of Kac and Moerbeke. His quantization conditions are similar to Bethe ansatz equations. We have studied the quantum periodic Toda lattice by the direct diagonalization of the Hamiltonian and shown that eigenvalues satisfy Gutzwiller's quantization conditions [8, 9]. Meanwhile, Sklyanin [10] has combined the quantum spectral transform method (QSTM) (R-matrix method) and Gutzwiller's formulation and derived an equation for the spectrum of the quantum Toda lattice.

In the pioneering work on the quantum Toda lattice, Sutherland [11] has shown that quantum particle (hole) excitations correspond to classical solitons (phonons) respectively. Since then there have been many studies looking for the classicalquantum correspondence in integrable systems. Most of them rely on the similarity of the classical and quantum dispersion relations. Mertens and Hader [12] assumed the validity of Bethe ansatz equations for the Toda lattice and obtained the dispersion relations of low energy excitations. We have studied thermodynamics of the quantum Toda lattice by combining Gutzwiller's quantization conditions and Yang-Yang's thermodynamic formulation [13]. Siddharthan and Shastry [14] have carried out comprehensive study of the quantum Toda lattice based on Gutzwiller's quantization conditions. Especially they discussed the relation between quantum 
numbers and properties of excited states. Recently Schliemann and Mertens [15] have applied the coherent state description to the Toda lattice and estimated the decoherence length of a solitary solution. In the case of integrable models of pairwise interactions, those authors $[16,17]$ have applied collective field theory [18] to nonlinear Schrödinger model and Calogero-Sutherland model, and derived solitonic density profiles and dispersions due to particle excitations. Sutherland and Campbell [19] have investigated classical solitons of Calogero-Sutherland model and their correspondence with the quantum system.

Soliton is spatially localized classical object, therefore, if we look for solitonic objects in quantum mechanics, we should investigate spatial properties of quantum states by some measures. Although Bethe ansatz equations give us energy spectra and dispersion relations, the calculation of eigenfunctions is very involved for manyparticle system. Recently there have been some mathematical developments on the wave function of the quantum Toda lattice [20, 21]. However, practical numerical calculation of the eigenfunction is still computationally difficult work. Furthermore there is no trace where solitonic objects come out in quantum wave functions. In order to look for geometric objects, we will define the notion of the most probable configuration (MPC) of eigenstates. Then it will be shown that the MPC of the excited state of the quantum Toda lattice has either solitonic or phonon-like profile depending on particle or hole excitations respectively. Our main emphasis will be on the validity of the MPC for the study of solitonic objects in quantum mechanics.

In section 2, we will describe the classical-quantum correspondence based on the discriminant of the system. The wave functions of the Toda lattice are reviewed in section 3, where hard-wall and harmonic chain limits are discussed in some detail. Section 4 deals with the notion and the validity of the most probable configurations and the practical way of estimating them. Numerical results will be presented in section 5 , and section 6 is devoted to a summary. 


\section{CLASSICAL-QUANTUM CORRESPONDENCE}

In this section, we will describe the correspondence between classical and quantum mechanics of the Toda lattice. The key observation is that the discriminant defined by the characteristic polynomial of the periodic Jacobi matrix associated with Lax form plays fundamental role both in classical and quantum mechanics. In a recent work, Siddharthan and Shastry [14] have discussed in detail the correspondence between quantum numbers given by Bethe ansatz equations and the classical discriminant of the Lax matrix. For the purpose of later discussion, let us briefly summarize their arguments and some earlier works.

In classical mechanics, the discriminant $\Delta(\lambda)$ is given by

$$
\Delta(\lambda)=\operatorname{det}(2 \lambda I-2 L)+2=\sum_{i} A_{i}(2 \lambda)^{N-i},
$$

where $L$ is the Lax matrix in the Lax form $\frac{d L}{d t}=[B, L]$ of the periodic Toda lattice [3]. Since the eigenvalues of the matrix $L$ are constants of motion, the coefficients $A_{i}$ of the characteristic polynomial $\Delta(\lambda)$ are also constants of motion. In the formulation of Kac and Moerbeke [5], the auxiliary spectra $\mu_{i}$ are defined by the eigenvalues of the reduced matrix $L^{*}$ which is the $(N-1) \times(N-1)$ matrix given by removing the first row and the first column from the matrix $L$. They are confined in $N-1$ intervals satisfying $|\Delta(\lambda)| \geq 2$. The curve $\Delta(\lambda)$ defines hyperelliptic structure with $N-1$ slits on the Riemann sheet.

Although general initial value problems can be solved in terms of the generalized Riemann's theta function [6], one would like to know the behaviour of the solution in more intuitive way. In Ref.[22], Ferguson, Flaschka and McLaughlin studied the correspondence between the discriminant $\Delta(\lambda)$ and the classical solutions in detail and found the following rules.

Rule 1: A small amplitude harmonic normal mode excites essentially only one Toda action variable. Mode $j$ opens the $j$-th gap and the open gap is located inside the interval $|\lambda|<1$.

Rule 2: A single localized soliton in a periodic Toda lattice corresponds to a single excited action variable. The one open gap lies outside the interval $|\lambda|<1$.

These are very simple rules which relate the behaviour of the discriminant $\Delta(\lambda)$ to phonon/soliton excitations. The discriminants $\Delta(\lambda)$ of phonon/soliton excitations are illustrated in Fig.1.

The semiclassical quantization can be carried out by the EBK (Einstein-BrillouinKeller) formulation. The action integral $I_{i}[23]$ can be replaced by the substitutions $I_{i} \rightarrow\left(n_{i}+1 / 2\right) h$, where $n_{i}$ is an integer quantum number and the Maslov index is 2 for the Toda lattice. The action $I_{i}$ geometrically represents the area $\left|\Delta\left(\mu_{i}\right)\right| \geq 2$, and a large action $I_{i}$ corresponds to a large interval [14].

In quantum mechanics, Gutzwiller [7] has developed a systematic way of constructing the simultaneous eigenfunction of the conserved operators $\hat{H}$ and $\hat{A}_{i}$, and Pasquier and Gaudin [24] have extended his method generally for the $N$-particle periodic Toda lattice by employing the transfer matrix method. Gutzwiller's quantization conditions are rather involved and one should refer to his original works [7]. In the quantization procedure, the function $D(\kappa)$, which is defined in terms of 
eigenvalues $\left(E, A_{3}, A_{4}, \ldots, A_{N}\right)$ of conserved operators,

$$
\begin{aligned}
D(\kappa)= & (\hbar \kappa)^{N}+E(\hbar \kappa)^{N-2}-i A_{3}(\hbar \kappa)^{N-3}+A_{4}(\hbar \kappa)^{N-4} \\
& +i A_{5}(\hbar \kappa)^{N-5}+\cdots+i^{N} A_{N}
\end{aligned}
$$

plays fundamental role. Apparently the function $D(\kappa)$ is the quantum analogue of the classical characteristic polynomial of the Lax matrix and has a relationship $D(2 i x)=i^{N} \Delta(\hbar x)$. In the large $N$ limit, it was shown $[13,25]$ that Gutzwiller's quantization conditions are largely simplified and they become ordinary Bethe ansatz equations,

$$
\begin{aligned}
\phi_{j} & =\frac{1}{2} L \lambda_{j}+N \lambda_{j} \ln \hbar+\sum_{k} \arg \left(\Gamma\left(1+i\left(\lambda_{j}-\lambda_{k}\right)\right)\right) \\
& =\frac{m \pi}{N} \quad(\bmod \pi) .
\end{aligned}
$$

The common integer $m$ of RHS characterizes the symmetric property of the eigenfunction [9]. The rapidities (pseudo-momenta) $\left\{\lambda_{j}\right\}$ are approximately given by the zeros of the equation $D(i \lambda)=0$. The integer quantum numbers $\left\{n_{j}\right\}$ defined by $\phi_{j+1}-\phi_{j}=\pi\left(n_{j}+1\right)[14,26]$ represent the intervals of rapidities $\left\{\lambda_{j}\right\}$. The distributions of rapidities are essentially the same as one-dimensional free Fermi gas. The consecutive sequence of rapidities represents the ground state, while particle/hole excited sequences correspond to excited quantum states. Therefore the simple way to express an eigenstate is the sequence of integer quantum numbers $\left\{n_{j}\right\}$. For example, the ground state is represented by $(0,0, \ldots, 0)$, and lowest excitations are $(1,0, \ldots, 0)$ and $(0, \ldots, 0,1)$ where the outermost rapidity is excited. It is clear that those states which have reversed sequence are generally degenerate. The large quantum number $n_{j}$ corresponds to the large interval $\Delta \lambda_{j}=\lambda_{j+1}-\lambda_{j}$, and thus large action $I_{j}$ in classical mechanics [14].

Now the relationship between classical, semiclassical and quantum mechanics is evident. In Fig.2, we show three cases, i.e., ground state, hole-excited state and particle-excited state respectively. One can easily expect that the particle/hole excitations corresponding to soliton/phonon excitations in classical mechanics should have some exceptional properties in quantum mechanics. We would look for some soliton/phonon-like configurations in quantum mechanics in the subsequent sections. 


\section{WAVE FUNCTIONS}

In this section we will briefly review the wave function of the quantum Toda lattice. The Hamiltonian of the $N$-particle Toda lattice in a box with length $L$ is

$$
\hat{H}=\frac{1}{2} \sum_{i=1}^{N} p_{i}^{2}+\sum_{i=1}^{N} \exp \left\{\left(x_{i}-x_{i+1}\right) / a\right\},
$$

where $a$ is the range of the potential, and we set the units such that the mass $m=1$ and the potential strength $V_{0}=1$. We will impose the periodic boundary condition $x_{N+1}=x_{1}+L$ so that the particles are confined in a ring with circumference $L$. In order to make the potential term in a symmetric form, let us transform the coordinates as follows

$$
y_{i}=x_{i}+(N-2 i+1) \frac{L}{2 N} .
$$

Then the Hamiltonian becomes an ordinary form of the periodic Toda lattice

$$
\hat{H}=-\frac{\hbar^{2}}{2} \sum_{i=1}^{N} \frac{\partial^{2}}{\partial y_{i}^{2}}+e^{-1 / D a} \sum_{i=1}^{N} \exp \left\{\left(y_{i}-y_{i+1}\right) / a\right\}
$$

with $y_{N+1}=y_{1}$ and $D=N / L$.

One of the profitable features of the Toda lattice is that it reduces to a simple model in an appropriate limit. It becomes the hard-wall potential (extreme anharmonic case) in the $a \rightarrow 0$ limit, while it reduces to the harmonic chain in the $a \rightarrow \infty$ limit. These limiting cases are well known and discussed in detail in Ref. [14] where their harmonic limit (strongly quantum anharmonic limit) corresponds to our $a \rightarrow \infty(a \rightarrow 0)$ limit respectively. It is expected that the Toda lattice with parameter $0<a<\infty$ will have properties between these two limits.

\subsection{Symmetric properties}

In Ref. [9] we have shown that the Hamiltonian of the Toda lattice has the symmetry of dihedral group $D_{N}$ which represents the symmetry of $N$-sided regular polygon. The dihedral group $D_{N}$ consists of $2 N$ elements $s^{i} t^{j}(0 \leq i \leq N-1, j=$ $0,1)$, where shift operator $s$ and inversion operator $t$ are generators transforming the coordinates as

$$
s y_{i}=y_{i+1}, \quad t y_{i}=-y_{N+1-i},
$$

and satisfy the relations

$$
s^{N}=1, \quad t^{2}=1, \quad t s t^{-1}=s^{-1} .
$$

The eigenstates are classified according to the irreducible representations of the dihedral group. They are labeled by $A_{1}, A_{2}, E_{k}(1 \leq k \leq(N-1) / 2)$ for $N=$ odd and $A_{1}, A_{2}, B_{1}, B_{2}, E_{k}(1 \leq k \leq(N-2) / 2)$ for $N=$ even. The $A_{1} / A_{2}$ and $B_{1} / B_{2}$ states are generally degenerate and we will simply call them $A(B)$ -symmetric state. $A$ or $B$ representation is 1-dimensional, while $E_{k}$ representation 
is 2-dimensional. The eigenfunctions are transformed under the operators $s$ and $t$ as

$$
\begin{array}{ccc}
s \Phi=\Phi, & t \Phi=\Phi^{*}, & \text { for } A-\text { symmtery } \\
s \Phi=-\Phi, & t \Phi=-\Phi^{*}, & \text { for } B \text { - symmtery } \\
s\left(\begin{array}{c}
\Phi \\
\Phi^{*}
\end{array}\right)=\left(\begin{array}{c}
e^{i 2 \pi k / N} \Phi \\
e^{-i 2 \pi k / N} \Phi^{*}
\end{array}\right), t\left(\begin{array}{c}
\Phi \\
\Phi^{*}
\end{array}\right)=\left(\begin{array}{c}
\Phi^{*} \\
\Phi
\end{array}\right), & \text { for } E_{k} \text { - symmtery } .
\end{array}
$$

One should note that the integer $m$ defined by Eq.(2.3) has a one-to-one correspondence to the irreducible representations of the dihedral group $D_{N}[9]$.

\subsection{Hard-wall potential $(a=0)$}

The zero range limit $(a \rightarrow 0)$ corresponds to the hard-wall (h.w.) potential, i.e., the potential term becomes

$$
\exp \left\{\left(x_{i}-x_{i+1}\right) / a\right\} \rightarrow V_{\mathrm{hw}}\left(x_{i}-x_{i+1}\right)=\left\{\begin{aligned}
\infty, & \text { for } x_{i}>x_{i+1} \\
0, & \text { for } x_{i} \leq x_{i+1}
\end{aligned}\right.
$$

The particles stay always in a definite order $x_{1}<x_{2}<\cdots<x_{N}$. Therefore the particle motions in the hard-wall potentials are equivalent to those of free fermions or hard-core bosons if the configuration space is restricted in the region $x_{1}<x_{2}<\cdots<x_{N}$. Thus the wave function is

$$
\Phi\left(x_{1}, x_{2}, \ldots, x_{N}\right)=\left(N L^{N}\right)^{-1 / 2} \operatorname{det}\left\{\exp \left(\frac{2 \pi i n_{j} x_{j^{\prime}}}{L}\right)\right\}_{1 \leq j, j^{\prime} \leq N}
$$

$N$ different integers $\left\{n_{j}\right\}\left(n_{1}<n_{2}<\cdots<n_{N}\right)$ are quantum numbers. Note that a common shift of each quantum number $n_{j}$ only changes center-of-mass motion and does not affect intrinsic motion. Thus the intrinsic wave function depends only on the intervals $\left\{\Delta n_{j}\right\}$ of the quantum numbers. If $\Delta n_{j}$ is defined by $\Delta n_{j}=$ $n_{j+1}-n_{j}-1$, then $N-1$ intrinsic quantum numbers $\left\{\Delta n_{j}\right\}$ are exactly equivalent to the quantum numbers $\left\{n_{j}\right\}$ defined in section 2 .

The ground state is represented by the quantum numbers $\Delta n_{1}=\Delta n_{2}=\cdots=0$ and the energy is $E_{0}=\frac{1}{12} N\left(N^{2}-1\right) \epsilon_{0}$ with $\epsilon_{0}=\frac{\hbar^{2}}{2}\left(\frac{2 \pi}{L}\right)^{2}$ in the C.M. system. The excitation energy can be calculated for low energy excitations. Typical excitations are illustrated in Fig.3. For the 1-hole (1h) or 1-particle (1p) excitations with $m$-th hole (particle) excited, the excitation energies are [14]

$$
\begin{aligned}
\Delta E(1 \mathrm{~h}) / \epsilon_{0} & =\frac{m}{N}(N+1)(N-m), \\
\Delta E(1 \mathrm{p}) / \epsilon_{0} & =\frac{m}{N}(N-1)(N+m) .
\end{aligned}
$$

For the 1 h1p-excitation with $m_{1}$-th hole jumping to $m_{2}$-th particle $\left(m_{1} \geq 1, m_{2} \geq\right.$ 1), the excitation energy is

$$
\begin{aligned}
\Delta E(1 \mathrm{~h} 1 \mathrm{p}) / \epsilon_{0} & =\frac{m}{N}(N-1)(N+m)-2 m\left(m_{1}-1\right) \\
m & =m_{1}+m_{2}-1 .
\end{aligned}
$$


For more complicated $2 \mathrm{~h} 2 \mathrm{p}$-excitation where $m_{1}$-th and $m_{1}^{\prime}$-th holes are removed to $m_{2}$-th and $m_{2}^{\prime}$-th particles $\left(m_{1}^{\prime}>m_{1} \geq 1, m_{2}^{\prime}>m_{2} \geq 1\right)$, the excitation energy is

$$
\begin{aligned}
\Delta E(2 \mathrm{~h} 2 \mathrm{p}) / \epsilon_{0}= & m\left(N+1-\frac{m}{N}\right)+\left(m_{1}+m_{2}-1\right)\left(m_{2}-m_{1}-1\right) \\
& +\left(m_{1}^{\prime}+m_{2}^{\prime}-1\right)\left(m_{2}^{\prime}-m_{1}^{\prime}-1\right) \\
m= & m_{1}+m_{2}+m_{1}^{\prime}+m_{2}^{\prime}-2 .
\end{aligned}
$$

One can obtain the symmetry of an eigenstate by applying $s$ and $t$ operators on the wave function (3.8)

$$
s \Phi=e^{ \pm i 2 \pi m_{\mathrm{s}} / N} \Phi, \quad t \Phi \propto \Phi^{*}
$$

where $m_{\mathrm{s}}$ is defined by the steps which are necessary to reach the excited pattern from the ground state. One step means that an occupied site is moved to its nearest neighbour vacant site. It turns out that the steps $m_{\mathrm{s}}$ is equal to $m$ defined by (3.9-3.14) in the cases of $1 \mathrm{~h}, 1 \mathrm{p}, 1 \mathrm{~h} 1 \mathrm{p}$ and $2 \mathrm{~h} 2 \mathrm{p}$ states. By comparing (3.15) with the transformation rule (3.6), it is recognized that the state with $m_{\mathrm{s}}$-steps has $E_{k}$-symmetry with $k=m_{\mathrm{s}}(\bmod N)$. In the case of $k=0(N / 2)$, the state has $A(B)$-symmetry.

\subsection{Harmonic chain $(a \rightarrow \infty)$}

At $a \rightarrow \infty$, the potential term can be approximated by the quadratic form and the Toda lattice reduces to the harmonic chain. Therefore the wave function of the harmonic chain is given by the well-known harmonic oscillator (h.o.) wave functions. Let us describe them in some detail, since we will use those wave functions as trial variational bases for the Toda lattice with finite $a$.

The Hamiltonian of the harmonic chain (h.c.) is in a dimensionless form

$$
\hat{H}_{\mathrm{hc}}=\frac{1}{2} \sum_{i=1}^{N} p_{i}^{2}+\frac{1}{2} \sum_{i=1}^{N}\left(y_{i}-y_{i+1}\right)^{2} .
$$

After making the potential term in a canonical quadratic form, one can obtain the Hamiltonian in the normal coordinates $\left\{\eta_{i}, \xi_{i}\right\}(0 \leq i \leq N-1)$

$$
\hat{H}_{\mathrm{hc}}=\frac{1}{2} \sum_{i=0}^{N-1} \eta_{i}^{2}+\frac{1}{2} \sum_{i=0}^{N-1} \omega_{i}^{2} \xi_{i}^{2}
$$

with $\omega_{k}=2 \sin \frac{k \pi}{N} \quad(0 \leq k \leq N-1)$, and $\omega_{0}=0$ corresponds to the free motion of the center-of-mass. Since $\omega_{N-k}=\omega_{k}$, normal modes are doubly degenerate except for $\omega_{N / 2}$ in $N=$ even case. The linear transformation is $y_{i}=\sum_{j} T_{i j} \xi_{j}, p_{i}=$ $\sum_{j} T_{i j} \eta_{j}$ with the orthogonal matrix $T$

$$
T=\left[\boldsymbol{v}_{0}, \boldsymbol{v}_{1}^{(1)}, \boldsymbol{v}_{1}^{(2)}, \ldots,\left\{\boldsymbol{v}_{N / 2}\right\}\right]
$$

where $\boldsymbol{v}_{k}^{(1)}$ and $\boldsymbol{v}_{k}^{(2)}$ are eigenvectors for the degenerate eigenmode $\omega_{k}(1 \leq k \leq$ $\left.\left[\frac{N-1}{2}\right]\right)$. Terms in $\{\cdots\}$ should be understood to be present only for $N=$ even case 
hereafter. Their components are

$$
\left(\boldsymbol{v}_{k}^{(1)}\right)_{j}=\sqrt{\frac{2}{N}} \cos \frac{2 k(j-1) \pi}{N}, \quad\left(\boldsymbol{v}_{k}^{(2)}\right)_{j}=\sqrt{\frac{2}{N}} \sin \frac{2 k(j-1) \pi}{N}, \quad 1 \leq j \leq N .
$$

The eigenvector $\boldsymbol{v}_{0}$ corresponds to the C.M. motion $\omega_{0}=0$ and its components are $\left(\boldsymbol{v}_{0}\right)_{j}=1 / \sqrt{N}$. The column $\boldsymbol{v}_{N / 2}$ is present only for $N=$ even and non-degenerate, and its components are $\left(\boldsymbol{v}_{N / 2}\right)_{j}=(-1)^{j-1} / \sqrt{N}$.

The operators $s$ and $t$ change the normal coordinate $\left\{\xi_{i}\right\}=\left(\xi_{0}, \xi_{1}^{(1)}, \xi_{1}^{(2)}, \ldots,\left\{\xi_{N / 2}\right\}\right)$ as

$$
\begin{aligned}
& s\left(\begin{array}{c}
\xi_{k}^{(1)} \\
\xi_{k}^{(2)}
\end{array}\right)=\left(\begin{array}{cc}
\cos \frac{2 k \pi}{N} & \sin \frac{2 k \pi}{N} \\
-\sin \frac{2 k \pi}{N} & \cos \frac{2 k \pi}{N}
\end{array}\right)\left(\begin{array}{l}
\xi_{k}^{(1)} \\
\xi_{k}^{(2)}
\end{array}\right), \quad s \xi_{0}=\xi_{0}, \quad\left\{s \xi_{N / 2}=-\xi_{N / 2}\right\}, \\
& t\left(\begin{array}{c}
\xi_{k}^{(1)} \\
\xi_{k}^{(2)}
\end{array}\right)=\left(\begin{array}{cc}
-\cos \frac{2 k \pi}{N} & \sin \frac{2 k \pi}{N} \\
\sin \frac{2 k \pi}{N} & \cos \frac{2 k \pi}{N}
\end{array}\right)\left(\begin{array}{l}
\xi_{k}^{(1)} \\
\xi_{k}^{(2)}
\end{array}\right), \quad t \xi_{0}=-\xi_{0}, \quad\left\{t \xi_{N / 2}=\xi_{N / 2}\right\} .
\end{aligned}
$$

The intrinsic wave function of the harmonic chain (3.17) is a product of the h.o. wave functions. We use 2-dimensional h.o. wave functions for the degenerate eigenmodes $\omega_{k}=\omega_{N-k}$, and 1-dimensional h.o. wave function for the non-degenerate eigenmode $\omega_{N / 2}$. The Hamiltonian of the 2-dimensional h.o. in the polar coordinate $(r, \theta)$ is in a dimensionless form

$$
\hat{H}_{\mathrm{ho}}=-\frac{1}{2}\left(\frac{\partial^{2}}{\partial r^{2}}+\frac{1}{r} \frac{\partial}{\partial r}+\frac{1}{r^{2}} \frac{\partial^{2}}{\partial \theta^{2}}\right)+\frac{1}{2} \omega^{2} r^{2} .
$$

The eigenstates are specified by quantum numbers $(n, \ell), n=0,1,2, \ldots, l=$ $0, \pm 1, \pm 2, \ldots$ The energy is $E_{n \ell}=(2 n+|\ell|+1) \omega$ and the wave function is written as

$$
\begin{aligned}
\phi_{n \ell}(\omega ; r, \theta) & =R_{n \ell}(\omega ; r) \Theta_{\ell}(\theta) \\
R_{n \ell}(\omega ; r) & =N_{n \ell}(\sqrt{\omega} r)^{|\ell|} L_{n}^{|\ell|}\left(\omega r^{2}\right) \exp \left(-\frac{1}{2} \omega r^{2}\right) \\
\Theta_{\ell}(\theta) & =\frac{1}{\sqrt{2 \pi}} e^{i \ell \theta}
\end{aligned}
$$

with the normalization $N_{n \ell}^{2}=2 \omega n ! /(n+|\ell|)$ ! and $L_{n}^{\ell}(x)$ is the Laguerre polynomial. The 1-dimensional h.o. wave function corresponding to the energy $E_{n}=(n+1 / 2) \omega$ is

$$
\varphi_{n}(\omega ; x)=N_{n} H_{n}(\sqrt{\omega} x) \exp \left(-\frac{1}{2} \omega x^{2}\right)
$$

with the normalization $N_{n}^{2}=\sqrt{\omega} /\left(2^{n} n ! \sqrt{\pi}\right)$ and $H_{n}(x)$ is the Hermite polynomial.

The intrinsic wave function is a product of 2-dimensional h.o. wave functions and, in the case of $N=$ even, one 1-dimensional h.o. wave function. By making 
coordinate transformations $\xi_{k}^{(1)}=r_{k} \cos \theta_{k}, \xi_{k}^{(2)}=r_{k} \sin \theta_{k}$, total wave function $\Phi$ can be expressed as

$\Phi\left(r_{1}, \theta_{1}, r_{2}, \theta_{2}, \ldots,\left\{\xi_{N / 2}\right\}\right)=\phi_{n_{1} \ell_{1}}\left(\omega_{1} ; r_{1}, \theta_{1}\right) \phi_{n_{2} \ell_{2}}\left(\omega_{2} ; r_{2}, \theta_{2}\right) \ldots \times\left\{\varphi_{n_{N / 2}}\left(\omega_{N / 2} ; \xi_{N / 2}\right)\right\}$.

Let us examine the symmetry of eigenstates. From Eq.(3.20,3.21), one can obtain the transformations of the polar coordinates under the symmetry operators $s$ and $t$ as

$$
s\left(\begin{array}{c}
r_{k} \\
\theta_{k}
\end{array}\right)=\left(\begin{array}{c}
r_{k} \\
\theta_{k}-\frac{2 k \pi}{N}
\end{array}\right), \quad t\left(\begin{array}{c}
r_{k} \\
\theta_{k}
\end{array}\right)=\left(\begin{array}{c}
r_{k} \\
-\theta_{k}-\frac{2 k \pi}{N}+\pi
\end{array}\right) .
$$

One should note that the symmetry operators only change angle variables. Thus the transformations of the wave function $\Phi$ are

$$
\begin{aligned}
& s \Phi=\exp \left[i \sum_{k}\left(-\frac{2 \pi k \ell_{k}}{N}\right)\right]\left\{(-1)^{n_{N / 2}}\right\} \Phi, \\
& t \Phi=\exp \left[i \sum_{k}\left(\pi-\frac{2 \pi k}{N}\right) \ell_{k}\right] \Phi^{*} .
\end{aligned}
$$

The complex conjugate wave function $\Phi^{*}$ is

$$
\begin{aligned}
& \Phi^{*}\left(r_{1}, \theta_{1}, \ldots,\left\{\xi_{N / 2}\right\} ; n_{1}, \ell_{1}, n_{2}, \ell_{2}, \ldots,\left\{n_{N / 2}\right\}\right) \\
& \quad=\Phi\left(r_{1}, \theta_{1}, \ldots,\left\{\xi_{N / 2}\right\} ; n_{1},-\ell_{1}, n_{2},-\ell_{2}, \ldots,\left\{n_{N / 2}\right\}\right),
\end{aligned}
$$

and thus $\Phi$ and $\Phi^{*}$ are degenerate unless $\ell_{1}=\ell_{2}=\cdots=0$. Then the symmetry of a state can be read off by the following formulas.

For $N=$ odd,

$$
\sum_{k} k \ell_{k}=\left\{\begin{array}{rrl}
0 & (\bmod N) & \text { for } A-\text { symmetry } \\
\pm m & (\bmod N) & \text { for } E_{m}-\text { symmetry }
\end{array}\right.
$$

For $N=$ even,

$$
\sum_{k} k \ell_{k}+\frac{N}{2} n_{N / 2}=\left\{\begin{array}{rll}
0 & (\bmod N) & \text { for } A-\text { symmetry } \\
N / 2 & (\bmod N) & \text { for } B-\text { symmetry } \\
\pm m & (\bmod N) & \text { for } E_{m}-\text { symmetry }
\end{array}\right.
$$

By appropriately multiplying a phase factor, one can recover the transformation rule (3.6).

\subsection{Toda lattice $(0<a<\infty)$}

The difficulty of the Toda lattice is that no explicit formulas are available for the spectrum or the eigenfunction. This situation contrasts with other well-known integrable models like Bose gas with $\delta$-function interaction or Calogero-Sutherland model. Although Gutzwiller's quantization conditions are well defined and searching the eigenvalues of the Hamiltonian and conserved quantities can be carried out 
for small particle numbers, e.g. $N \leq 10$, the calculation of the eigenfunction is computationally hard work. Recently an integral representation of the eigenfunction has been constructed [21], however, it is not very useful in numerical calculation since one has to carry out multi-dimensional integration. Therefore it is more practical to employ ordinary variational calculations which give us eigenfunctions as well as eigenvalues simultaneously.

In Ref. [9] we have diagonalized the Hamiltonain by employing the bases composed of the product of 1-dimensional harmonic oscillators. By appropriately symmetrizing the bases, we could calculate eigenvalues of the conserved quantities $\hat{A}_{i}$ as well as the energy. Now we will specify the eigenstates by integer quantum numbers $\left\{n_{j}\right\}$ instead of the eigenvalues of $\hat{H}$ and $\hat{A}_{i}$. Thus the eigenvalues of the conserved quantities $\hat{A}_{i}$ are not necessary. Therefore we will choose basis functions such that we can treat more easily from the viewpoint of symmetric property. In fact we have employed the eigenfunctions of the harmonic chain described in section 3 -3. Since the symmetric property, i.e., invariance under the dihedral group $D_{N}$, is the same as the Toda lattice, those eigenfunctions of the harmonic chain have the $D_{N}$ symmetry from the beginning, and further symmetrization of the basis is not necessary.

The details of the calculation of the matrix elements are involved and they are postponed to Appendix A. 


\section{MOST PROBABLE CONFIGURATIONS}

Low energy excitations are usually studied by quantizing small amplitude fluctuations around classical configuration. They are harmonic vibrations and are expressed by sinusoidal waves. The classical soliton solution of the Toda lattice suggests that the configurations of low energy excited states in quantum mechanics are different from sinusoidal form due to nonlinear interactions, and thus, small harmonic vibration expansion can not be applied. Therefore we will study low energy excited states by looking into the exact wave functions of the Toda lattice.

In order to investigate excited states configurations, we will employ the most probable configuration (MPC) which seems good measure to estimate solitonic configuration in many-coordinates space. The MPC $\left\{x_{i}^{0}\right\}$ is defined by the coordinates which maximize the probability density $P\left(\left\{x_{i}\right\}\right)=\left|\Phi\left(\left\{x_{i}\right\}\right)\right|^{2}$, where $\Phi\left(x_{1}, x_{2}, \ldots, x_{N}\right)$ is the wave function of the $N$-particle Toda lattice, i.e.,

$$
\left(\frac{\partial|\Phi|^{2}}{\partial x_{i}}\right)_{\left\{x_{i}^{0}\right\}}=0 .
$$

The MPC $\left\{x_{i}^{0}\right\}$ can be calculated for the hard-wall $(a=0)$ and the harmonic chain $(a \rightarrow \infty)$ cases in analytical way. However, one has to carry out some numerical calculations for $0<a<\infty$.

Although the MPC $\left\{x_{i}^{0}\right\}$ can be given either analytically or numerically, one would naturally wonder whether those configurations around $\left\{x_{i}^{0}\right\}$ exhaust the probability, in other words, the probability $\left|\Phi\left(\left\{x_{i}\right\}\right)\right|^{2} d V$ near the MPC is large enough to define the notion of MPC. The simplest estimation is just to integrate over the MPC $\left\{x_{i}^{0}\right\}$ and to see how large the probability is. In order to carry out the integration, we will approximate the wave function around the MPC by the Gaussian form, i.e.,

$$
\begin{aligned}
P\left(\left\{x_{i}\right\}\right) & =P\left(\left\{x_{i}^{0}\right\}\right)+\sum_{k, \ell} \frac{1}{2}\left(\frac{\partial^{2} P}{\partial x_{k} \partial x_{\ell}}\right)_{\left\{x_{i}^{0}\right\}} \Delta x_{k} \Delta x_{\ell} \\
& =P\left(\left\{x_{i}^{0}\right\}\right)\left(1+{ }^{t} \overrightarrow{\Delta x} H \overrightarrow{\Delta x}\right) \\
& \simeq P\left(\left\{x_{i}^{0}\right\}\right) \exp \left({ }^{t} \overrightarrow{\Delta x} H \overrightarrow{\Delta x}\right)
\end{aligned}
$$

with $\Delta x_{k}=x_{k}-x_{k}^{0}$, and the Hessian matrix $H$ is defined by

$$
H_{k \ell}=\frac{1}{2} P\left(\left\{x_{i}^{0}\right\}\right)^{-1}\left(\frac{\partial^{2} P}{\partial x_{k} \partial x_{\ell}}\right)_{\left\{x_{i}^{0}\right\}} .
$$

After diagonalizing the Hessian matrix, one can approximately calculate the probability around the $\operatorname{MPC}\left\{x_{i}^{0}\right\}$ as

$$
I_{\mathrm{MPC}}=\int P\left(\left\{x_{i}\right\}\right) d V \simeq P\left(\left\{x_{i}^{0}\right\}\right) \prod_{k} \sqrt{\frac{\pi}{\left|h_{k}\right|}},
$$

where $\left\{h_{k}\right\}$ are eigenvalues of the Hessian matrix which are negative by definition. In the case of a small eigenvalue, one should be careful about the integration since the Gaussian approximation is questionable. 
Another estimation of the MPC is the average extension $\bar{r}$ of the MPC, which is related to the average $\bar{h}$ of $\left\{h_{k}\right\}$ as $\bar{r}=1 / \sqrt{\bar{h}}$. $\bar{h}$ is defined by the geometrical mean of $\left\{h_{k}\right\}$, i.e., $\bar{h}=\left(\prod_{k}\left|h_{k}\right|\right)^{1 / N}$, which seems preferable to the arithmetic mean $\bar{h}^{\prime}=\sum_{k}\left|h_{k}\right| / N$, since $I_{\mathrm{MPC}}$ is determined by the product of $\left\{h_{k}\right\}$ and there are large differences in the magnitudes of $h_{k}$.

In the following subsections, we will describe the $\operatorname{MPC}\left\{x_{i}^{0}\right\}$ in some detail.

\subsection{Hard-wall $(a=0)$}

In the case of hard-wall potential, one can calculate the $\operatorname{MPC}\left\{x_{i}^{0}\right\}$ analytically and examine how large the probability $I_{\mathrm{MPC}}$ is. Although the calculations are straightforward, they are very involved and details are described in Appendix B. Here let us briefly summarize the conclusions of Appendix B.

(1) The MPC $\varepsilon_{\mathrm{h}}\left(\varepsilon_{\mathrm{p}}\right)$ of the $E_{m}$-symmetric 1-hole (1-particle) excited state is given by

$$
\varepsilon_{\mathrm{h}}(x)=\sum_{n=1}^{m}(-1)^{n} \frac{1}{n \pi} \sin 2 n \pi x, \quad \varepsilon_{\mathrm{p}}(x)=-\varepsilon_{\mathrm{h}}(x) .
$$

$\varepsilon(x)(|x| \leq 1 / 2)$ is the continuum approximation of the deviation $\varepsilon_{i} / D=x_{i}^{0}-\bar{x}_{i}$ with $\bar{x}_{i}=i / D$ being the equidistance configuration corresponding to the ground state.

(2) Apart from the trivial zero-eigenvalue due to the translational invariance, one of the eigenvalues of the Hessian matrix is identically zero in the large $N$ limit. It is understandable since there are $N$ equivalent MPC's due to the $D_{N}$ symmetry and the distance between the nearest MPC's is $\sqrt{\frac{2}{N}} \frac{1}{D}$ as $N \rightarrow \infty$, thus the probability $|\Phi|^{2}$ does not change along the nearest MPC's. This direction is certainly the eigenvector of the zero-eigenvalue of the Hessian matrix.

(3) The average extension of the MPC is $\bar{r}=\frac{e}{\sqrt{2} \pi} \frac{1}{D} \simeq 0.61 / D$ irrespective of the symmetry. Therefore the extension of the MPC is, roughly speaking, about a half of the equilibrium spacing.

(4) The probability $I_{\mathrm{MPC}}$ turns out, in the Gaussian approximation,

$$
I_{\mathrm{MPC}}=\left(\frac{e}{\sqrt{2 \pi}}\right)^{N-1} \times \frac{m}{\exp \left(1+\frac{1}{2}+\frac{1}{3}+\cdots+\frac{1}{m}-1\right)}
$$

both for $1 \mathrm{~h}$ and $1 \mathrm{p}$ excitations in the case of $E_{m}$-symmetry. Since the normalization of the ground state is $\left(\frac{e}{\sqrt{2 \pi}}\right)^{N}$ in the same approximation, numerical ratio is close to 1 . Therefore the notion of the MPC is valid and the probability around MPC fills the probability.

\subsection{Harmonic chain $(a \rightarrow \infty)$}

One can also carry out analytic calculations for harmonic chain case [14]. The ground state of $E_{m}$-symmetry is a 1-hole state which has quantum numbers $n_{1}=$ $n_{2}=\cdots=0, \ell_{1}=\ell_{2}=\cdots=0$ except for $\ell_{m}= \pm 1$. Thus the probability density is

$$
|\Phi|^{2} \propto r_{m}^{2} \exp \left(-\sum_{k} \omega_{k} r_{k}^{2}\right)
$$


where $\omega_{k}=e^{-\frac{1}{2 D a}} \frac{1}{a} \times 2 \sin \frac{k \pi}{N} \simeq \frac{1}{a} \times 2 \sin \frac{k \pi}{N}$. The MPC is $r_{m}^{0}=1 / \sqrt{\omega_{m}}$ and $r_{k}^{0}=0$ for $k \neq m$, which corresponds to the normal coordinates,

$$
\xi^{0}=\left(0, \ldots, \xi_{m}^{(1)}=\frac{1}{\sqrt{\omega_{m}}} \cos \alpha, \xi_{m}^{(2)}=\frac{1}{\sqrt{\omega_{m}}} \sin \alpha, 0, \ldots, 0\right) .
$$

Then the original coordinates are

$$
y_{i}^{0}=\left(T \boldsymbol{\xi}^{0}\right)_{i}=\sqrt{\frac{2}{N}} \frac{1}{\sqrt{\omega_{m}}} \cos \left(\frac{2 \pi m}{N}(i-1)-\alpha\right),
$$

where the angle $\alpha$ is arbitrary and the MPC consists of continuous configurations. By integrating out the radial coordinates by the Gaussian approximation, one can obtain $I_{\mathrm{MPC}}=\sqrt{2 \pi} / e \simeq 0.92$, which indicates that the MPC is well defined and almost fills the probability.

The 1-particle state of $E_{m}$-symmetry corresponding to the solitonic excitation has quantum numbers $n_{1}=n_{2}=\cdots=0, \ell_{2}=\ell_{3}=\cdots=0$ except for $\ell_{1}= \pm m$. The calculation is similar to the 1-hole state and the normal coordinates maximizing the probability turn out

$$
\boldsymbol{\xi}^{0}=\left(\xi_{1}^{(1)}=\sqrt{\frac{m}{\omega_{1}}} \cos \alpha, \xi_{1}^{(2)}=\sqrt{\frac{m}{\omega_{1}}} \sin \alpha, 0, \ldots, 0\right),
$$

and the original coordinates are

$$
y_{i}^{0}=\left(T \boldsymbol{\xi}^{0}\right)_{i}=\sqrt{\frac{2}{N}} \sqrt{\frac{m}{\omega_{1}}} \cos \left(\frac{2 \pi}{N}(i-1)-\alpha\right) .
$$

The integral over the MPC is

$$
I_{\mathrm{MPC}}=\sqrt{2 \pi} m^{m+1 / 2} e^{-m} / m ! \simeq 1
$$

by Stirling's formula. Therefore the MPC is well peaked around $\left\{y_{i}^{0}\right\}$ for the harmonic chain.

In both cases of $1 \mathrm{~h}$ and $1 \mathrm{p}$ excitations, the MPC consists of continuous configurations. This fact means that, besides the zero-eigenvalue due to the translational invariance, one of the eigenvalues of the Hessian matrix is identically zero. The average extension turns out $\bar{r}=\sqrt{a}$. Since $y_{i}^{0}=O(\sqrt{a})$ as $N \rightarrow \infty$, the MPC $\left\{y_{i}^{0}\right\}$ and the average extension $\bar{r}$ increases in the same order $O(\sqrt{a})$ as $a \rightarrow \infty$.

\subsection{Toda lattice $(0<a<\infty)$}

For the general Toda lattice, we have performed numerical calculation. Since we have wave functions given by the variational calculation, we can numerically search the MPC $\left\{x_{i}^{0}\right\}$. In practice, we have employed the simplex method to find the MPC $\left\{x_{i}^{0}\right\}$ which maximizes the probability density $P\left(\left\{x_{i}\right\}\right)$. One should note that there are $N$ equivalent MPC due to the $D_{N}$ symmetry. Once the MPC $\left\{x_{i}^{0}\right\}$ was found, the Hessian matrix can be numerically calculated and the integral $I_{\mathrm{MPC}}$ is estimated to measure the extent of the localization of the wave function.

It is found that one of the eigenvalues of the Hessian matrix is almost zero. It is natural since the MPC's are continuously distributed in the large $N$ limit both 
in the hard-wall and the harmonic chain limits, and the Toda lattice should be in the same situation. Therefore the local maxima of the probability stretch like a mountain ridge. The direction of the ridge is certainly the eigenvector of the small eigenvalue. For the $1 p$ solitonic excitation, the small eigenvalue corresponding to the flat direction is always the smallest one. However, it is not necessarily the smallest for the $1 \mathrm{~h}$ phonon-like excitations, since there is a change of the structure of the MPC as the range parameter $a$ is varied. We have approximately calculated the integral along this flat direction in the same way as the hard-wall case, which is explained in Appendix B. Details will be discussed in the next section. 


\section{RESULTS AND DISCUSSION}

We have calculated the MPC up to $N=12$ Toda lattice. Although we are interested in the thermodynamic limit, i.e., $N, L \rightarrow \infty$ with $D=N / L=$ fixed, the general formula are not available except for the hard-wall potential $(a=0)$ and the harmonic chain $(a \rightarrow \infty)$ cases. One can naturally expect that those states with $0<a<\infty$ are just in between two limiting cases. The $N=12$ particle system seems to be large enough to extrapolate to $N \rightarrow \infty$, but still under control in numerical calculation. We have fixed the density as $D=N / L=1$, which sets the length scale. We further choose the constant $\hbar=1$ for simplicity. The qualitative behaviour of the system does not depend on the scale $\hbar$, although it changes numerical results quantitatively. Since we are mainly interested in relatively geometrical configurations, we are not concerned with those absolute magnitudes. In the end, the Hamiltonian becomes

$$
\hat{H}=-\frac{1}{2} \sum_{i=1}^{N} \frac{\partial^{2}}{\partial y_{i}^{2}}+e^{-1 / a} \sum_{i=1}^{N} \exp \left\{\left(y_{i}-y_{i+1}\right) / a\right\}
$$

The parameter $a$ changes the ratio of the potential range to the equilibrium spacing of the ground state, and we have studied the system by changing the range parameter $a$. We have calculated the MPC of 1-hole and 1-particle excited states in $E_{1}, E_{2}, E_{3}$ and $E_{4}$-symmetry, in other words, up to 4 -th 1-hole/particle excited states. In order to show the validity of the MPC, we have also studied $2 \mathrm{~h} 2 \mathrm{p}$ state in $E_{4}$-symmetry.

In our calculation, trial functions consist of harmonic chain wave functions and variational parameters are angular frequencies of the harmonic oscillator basis besides the total number of basis. We have tried several combinations of angular frequencies and found that the reasonable choice is that they are proportional to those of the harmonic chain, i.e., $r \times 2 \sin \frac{k \pi}{N}$, and good stability is achieved by changing one common proportionality parameter $r$. The number of basis is about 4900 depending on the symmetry, which gives us the accuracy of low energy levels within $0.1 \%$. This number of basis seems large enough if one is interested only in low energy levels. However, there is a structure change of the MPC for 1 h state and, thus, the wave functions are calculated as precisely as possible, since the variational wave functions are less accurate than the energy levels.

In the followings, we will present the results of (1) energy levels, (2) morphology of the MPC, (3) fittings in terms of elliptic theta function and (4) two-soliton state.

\subsection{Energy levels}

In Fig.4, we show low energy levels of $0 \leq a \leq 2$ for each symmetry. At $a=0$ (hard-wall) the energy levels are calculated by the formula Eq.(3.9-3.14), while at large $a$ energy levels tend to those of harmonic chain. There are minima at $0.4<a<1.0$ where typical Toda lattice nature comes out. In Table 1, we list integer quantum numbers $\left\{n_{j}\right\}$ defined by $\phi_{j+1}-\phi_{j}=\pi\left(n_{j}+1\right)$ in section 2 . Only one of a pair of degenerate quantum numbers is shown. The lowest state always corresponds to 1-hole state, while 1-particle state is pushed upward and buried in higher excited states, especially for $E_{4}$-symmetry. One can expect that 1-particle state is located in far above energy levels at $E_{m}$-symmetry with large $m$, and there 
are complicated states like $1 \mathrm{~h} 1 \mathrm{p}, 2 \mathrm{~h} 2 \mathrm{p}, \ldots$ excitations in between. There is an accidental level crossing of 1-particle state in $E_{4}$-symmetry where 1-particle state corresponds to the 6 -th level in a small $a$ region while it comes down to the 5 -th level as $a$ increases, and there is a crossing at $a \simeq 0.55$.

TABLE 1

Quantum numbers of low energy levels for each symmetry. For $E_{4}$ symmetry, the upper (lower) quantum numbers of 5-th level corresponds to the range $a \leq 0.55(a \geq 0.55)$ respectively.

\begin{tabular}{ccccc}
\hline Level & $E_{1}$ & $E_{2}$ & $E_{3}$ & $E_{4}$ \\
\hline 1 & $(0,0,0,0,0,0,0,0,0,0,1)$ & $(0,0,0,0,0,0,0,0,0,1,0)$ & $(0,0,0,0,0,0,0,0,1,0,0)$ & $(0,0,0,0,0,0,0,1,0,0,0)$ \\
2 & $(1,0,0,0,0,0,0,0,0,1,0)$ & $(0,0,0,0,0,0,0,0,0,0,2)$ & $(0,0,0,0,0,0,0,0,0,1,1)$ & $(0,0,0,0,0,0,0,0,1,0,1)$ \\
3 & $(1,0,0,0,0,0,0,0,0,0,2)$ & $(1,0,0,0,0,0,0,0,1,0,0)$ & $(0,0,0,0,0,0,0,0,0,0,3)$ & $(0,0,0,0,0,0,0,0,0,2,0)$ \\
4 & $(0,1,0,0,0,0,0,0,1,0,0)$ & $(1,0,0,0,0,0,0,0,0,1,1)$ & $(1,0,0,0,0,0,0,1,0,0,0)$ & $(0,0,0,0,0,0,0,0,0,1,2)$ \\
5 & $(2,0,0,0,0,0,0,0,1,0,0)$ & $(1,0,0,0,0,0,0,0,0,0,3)$ & $(1,0,0,0,0,0,0,0,1,0,1)$ & $(1,0,0,0,0,0,1,0,0,0,0)$ \\
& & & & $(0,0,0,0,0,0,0,0,0,0,4)$ \\
\hline
\end{tabular}

In the region of large $a$, e.g. $a>10$, we have noticed that there is a relationship between the Toda lattice quantum numbers $\left\{n_{j}\right\}$ and the harmonic chain quantum numbers $\left(n_{j}^{\prime}, \ell_{j}^{\prime}\right)$ as

$$
n_{j}^{\prime}=\min \left(n_{j}, n_{N-j}\right), \quad \ell_{j}^{\prime}= \pm\left(n_{j}-n_{N-j}\right),
$$

where $\left(n_{j}^{\prime}, \ell_{j}^{\prime}\right)$ are the quantum numbers of the major component of the harmonic chain. These correspondences have been already discovered by Siddharthan and Shastry [14]. In Fig.5, we show the decomposition of the wave function into harmonic chain bases in the case of $E_{4}$-symmetry for example. It is evident that a single component becomes overwhelming as the range $a$ increases.

\subsection{Morphology of the most probable configurations}

We will show the most probable configuration $\left\{x_{i}^{0}\right\}$ in terms of the deviations from the equidistant configuration, i.e., $\varepsilon_{i}=x_{i}^{0}-\bar{x}_{i}$ with $\bar{x}_{i}=i$. In Fig.6 the convergence of the MPC is indicated for 1-hole excitation as the particle number $N$ increases. The deviation of $i$-th particle is plotted at $x=(2 i-1) / 2 N$ and only half region $0 \leq x \leq 1 / 2$ is shown due to the symmetry $\varepsilon_{\mathrm{h}}(x)=-\varepsilon_{\mathrm{h}}(1-x)$. One can easily expect that, in the large $N$ limit, the configuration of MPC will converge to a smooth profile. We show in Fig.7 the MPC's of $N=12$ particles. Since $\left\{\varepsilon_{i}\right\}$ is an odd function, i.e., $\varepsilon_{N+1-i}=-\varepsilon_{i}$, we plot them in the range $1 \leq i \leq 6$. One should also note that there are $N$ equivalent MPC's which shifts Fig.7 by $1,2, \ldots, N$ due to the $D_{N}$ symmetry. Smooth curves are interpolations given by the Spline function.

From Fig. 7 it is readily recognized that there are structure changes in the MPC for 1 h excited states with $E_{m}$-symmetry $(m \geq 2)$. In the large $N$ limit, one can make a continuous approximation by setting $x=i / N(0 \leq x \leq 1)$. Then the 1 h excited MPC at $a=0$ is $\varepsilon_{\mathrm{h}}(x)=\sum_{n=1}^{m} \frac{(-1)^{n}}{n \pi} \sin 2 n \pi\left(x-\frac{1}{2}\right)=\sum_{n=1}^{m} \frac{1}{n \pi} \sin 2 n \pi x(0 \leq x \leq$ 1) which does not have nodes in $0<x<1 / 2$, while $\varepsilon_{\mathrm{h}}(x) \propto \sin 2 m \pi x$ for $a \rightarrow \infty$. Therefore there must exist some structure changes in between. 
In Fig.8, the MPC's are shown in a different way, i.e., deviations $\varepsilon_{i}$ are plotted with respect to the potential range $a$. It is clear that the morphology of $1 \mathrm{~h}$ excited MPC changes for $E_{2}, E_{3}$ and $E_{4}$-symmetries as the range $a$ increases.

The average extension $\bar{r}$ smoothly increases from $\bar{r}=0.61(a=0)$, and $\bar{r} \simeq \sqrt{a}$ for $a>3$ irrespective of the symmetry. The probability measure $I_{\mathrm{MPC}}$ turns out $I_{\mathrm{MPC}} \simeq 1$ for $1 \mathrm{p}$ excitation, while $I_{\mathrm{MPC}} \simeq 2$ for $1 \mathrm{~h}$ excitation. (In the case of $E_{1}$-symmetry, $I_{\mathrm{MPC}} \simeq 1$, since $1 \mathrm{~h}$ and $1 \mathrm{p}$ excitations are identical.) The MPC's form a ridge and the probability is nearly constant on it. In our approximation, the integral is usually overestimated since the probability is approximated by its maximum value along the flat direction. Thus the probability $I_{\mathrm{MPC}}$ may happen to exceed one. Numerical results indicate that the MPC is well defined and essentially fills the probability.

Let us make several comments on each state of symmetry.

\subsubsection{E E-state}

This is a special case in which 1-hole and 1-particle excitations are identical. However, one should assign the profile $\varepsilon_{\mathrm{p}}(x)=-\varepsilon_{\mathrm{h}}(x)$ in accordance with other symmetries. In Fig.7, we conveniently plot $\varepsilon_{\mathrm{p}}(x)$ by shifting a half period relative to $\varepsilon_{\mathrm{h}}(x)$. Thus the figures look like $\varepsilon_{i}$ (particle) $=-\varepsilon_{i+6}$ (hole). When the range $a$ becomes large, the MPC approaches to sin-curve which is the MPC of harmonic chain. Also the shape of the curve at $a=0$ would become sin-curve as $N$ increases.

It is not easy to imagine the wave function of the $N=12$ particle system which has 11 intrinsic coordinates. Thus it is instructive to display $E_{1}$-symmetric states of $N=3$ system. In Fig.9 we show the contour plots of the probability density $|\Phi|^{2}$ for several range parameters. Then one can realize how three MPC's make a ridge as $a$ increases. In the same way, for the case of $N=12$, the probability density $|\Phi|^{2}$ would make a ridge with 12 bumps in the 11 intrinsic coordinates configuration space.

\subsection{2. $E_{2}$-state}

In the $E_{2}$-symmetry, $1 \mathrm{p}$ state is the 2 -nd level. For the $1 \mathrm{~h}$ state, there is a critical $a$-value $a_{c} \simeq 1.2$ where the structure changes, i.e., at $a<a_{c}$ the deviation $\varepsilon_{\mathrm{h}}(x)$ tends to one side, while at $a>a_{c} \varepsilon_{\mathrm{h}}(x)$ is oscillating with wave length $1 / 2$ and approaches $\varepsilon_{\mathrm{h}}(x) \propto \sin 4 \pi x$. The numerical calculations indicate that the structure change happens sharply, but it is not discontinuous, i.e., there is a common crossing point of two ridges. However, this behaviour could be due to the finite $N$ effect and it is not clear what would happen in the large $N$ limit. The approximate calculation of $I_{\mathrm{MPC}}$ becomes largely overestimated near $a=a_{c}$. For the $1 \mathrm{p}$ state, there is no structure change and the shape becomes close to $\varepsilon_{\mathrm{p}}(x) \propto \sin 2 \pi x$ when the range $a$ becomes large. At $a=0, \varepsilon_{i}$ (particle) $\simeq-\varepsilon_{i+6}$ (hole), i.e., the large deviations of $1 \mathrm{~h}$ and $1 \mathrm{p}$ states are located in the opposite direction. When the range $a$ increases, $\sin 4 \pi x$ component overwhelms for 1 h state, while $\sin 4 \pi x$ component decreases for $1 \mathrm{p}$ state.

\subsection{3. $E_{3}$-state}

The $1 \mathrm{p}$ state is the 3 -rd level and there is a $1 \mathrm{~h} 1 \mathrm{p}$ state in between. For the $1 \mathrm{~h}$ state, the critical value is $a_{c} \simeq 0.65$. At $a<a_{c}$ the deviation $\varepsilon_{\mathrm{h}}(x)$ tends to one 
side, while at $a>a_{c} \varepsilon_{\mathrm{h}}(x)$ is oscillating with wave length $1 / 3$ and approaches $\varepsilon_{\mathrm{h}}(x) \propto \sin 6 \pi x$. The eigenvalue of the flat direction is not the smallest one but the 3 -rd one for $a<1$, and it gets down to the smallest for $a>1$. For the $1 \mathrm{p}$ state, there is no structure change and the shape becomes close to $\varepsilon_{\mathrm{p}}(x) \propto \sin 2 \pi x$ when $a$ increases.

\subsection{4. $E_{4}$-state}

The $1 \mathrm{p}$ state is 6 -th level at $a<0.55$ and 5 -th level at $a>0.55$, and there is a level crossing at $a \simeq 0.55$. Those levels in between are $1 \mathrm{~h} 1 \mathrm{p}$ or $2 \mathrm{~h} 2 \mathrm{p}$ excited states. For the $1 \mathrm{~h}$ state, the critical value is $a_{c} \simeq 0.5$. At $a>a_{c} \quad \varepsilon_{\mathrm{h}}(x)$ is oscillating with wave length $1 / 4$ and tends to approach $\varepsilon_{\mathrm{h}}(x) \propto \sin 8 \pi x$. The eigenvalue of the flat direction is the 3 -rd or 4 -th one for $a<6$, while it becomes the smallest for $a>6$. For the $1 \mathrm{p}$ state, there is no structure change.

\subsection{Fittings in terms of elliptic theta function}

The shape of the MPC looks like a profile of the cnoidal wave of the classical periodic Toda lattice. At $a \rightarrow \infty$ (harmonic chain) the configuration of the MPC is $\varepsilon_{\mathrm{h}}(x) \propto \sin 2 m \pi x$ for the $E_{m}$-symmetric 1-hole excitation and $\varepsilon_{\mathrm{p}}(x) \propto \sin 2 \pi x$ for the 1-particle excitation, and those configurations are the same as normal coordinates of the classical harmonic chain. This similarity between the MPC and the classical solution is naturally expected also for the Toda lattice.

At finite $a$, the MPC deviates from the trigonometric form, however, it is similar to the cnoidal wave described by the elliptic function. The elliptic function is a doubly periodic function and it approaches to the trigonometric function at the nome $q \rightarrow 0$ limit. The classical Toda lattice with one open gap corresponds to genus $g=1$ and its solution is expressed by the elliptic function. And classical one open gap corresponds to quantum 1-hole/particle excitation depending on the location of the gap. Therefore it is worth trying to fit the MPC in terms of the elliptic function. A brief review of the cnoidal wave of the classical Toda lattice is summarized in Appendix C.

The solution of the classical Toda lattice with one gap is

$$
Q_{n}(t)=\ln \left\{\theta_{0}\left(\frac{\alpha t+\beta}{2 K} \pm j \frac{n-1}{N}\right) / \theta_{0}\left(\frac{\alpha t-\beta}{2 K} \pm j \frac{n-1}{N}\right)\right\}
$$

where $\alpha$ and $\beta$ are determined by initial conditions or the values of conserved quantities. $K$ is the complete integral of the first kind of modulus $k$ which is again determined by initial conditions. A positive integer $j$ indicates the number of oscillations in one period. There is a relationship $N \beta=j K$. By appropriately setting the time $t$ such that the profile is an odd function, $Q_{n}$ becomes

$$
\begin{aligned}
Q_{n} & =\ln \left\{\theta_{0}\left(j\left(x_{n} \pm \frac{1}{2 N}\right)\right) / \theta_{0}\left(j\left(x_{n} \mp \frac{1}{2 N}\right)\right)\right\} \\
& = \pm \ln \left\{\theta_{0}\left(j\left(x_{n}+\frac{1}{2 N}\right)\right) / \theta_{0}\left(j\left(x_{n}-\frac{1}{2 N}\right)\right)\right\}
\end{aligned}
$$


where $x_{n}=-\frac{1}{2}+\frac{2 n-1}{2 N}$. If the lattice points $x_{n}$ are considered continuous, then we can define the function $f_{1}(x)$ as

$$
f_{1}(x ; j)=\ln \left\{\theta_{0}\left(j\left(x-\frac{1}{2 N}\right)\right) / \theta_{0}\left(j\left(x+\frac{1}{2 N}\right)\right)\right\}
$$

and the function $f_{2}(x ; j)$ which shifts $f_{1}(x ; j)$ by a half period,

$$
f_{2}(x ; j)=\ln \left\{\theta_{0}\left(j\left(x-\frac{1}{2 N}\right)-\frac{1}{2}\right) / \theta_{0}\left(j\left(x+\frac{1}{2 N}\right)-\frac{1}{2}\right)\right\} .
$$

Since the magnitude of the deviation $\varepsilon(x)$ depends on the scale of the constant $\hbar$, we try to fit the MPC in terms of a multiple of $f_{1}(x ; j)$ or $f_{2}(x ; j)$ depending on the shape, and to extract the nome $q$ of the elliptic function which represents the extent of deformation from the trigonometric function. $q=0$ corresponds to the trigonometric function of the harmonic chain, while $q=1$ corresponds to an extreme anharmonic case.

In Fig.10, we show an example of fittings of the MPC's of 1p state for each symmetry. Fitted values are further approximated by the functional form $q(a)=$ $c e^{-\kappa a}$. For $1 \mathrm{p}$ solitonic excitations, the fitted parameters are listed in Table 2. As can be expected, the nome $q$ is a decreasing function of $a$ and $q \rightarrow 0$ as $a \rightarrow \infty$. There are two regions where numerical values $q(a)$ are well fitted. For $0 \leq a \leq 1$, the decreasing rate $\kappa$ is different depending on the symmetry, while it does not change so much for $5 \leq a \leq 10$. These fittings seem reasonable and we can conclude that there exists a close similarity between the MPC's and the classical cnoidal waves.

\begin{tabular}{|c|c|c|c|c|}
\hline \multirow[b]{2}{*}{$E_{m}$} & \multicolumn{2}{|c|}{$0 \leq a \leq 1$} & \multicolumn{2}{|c|}{$5 \leq a \leq 10$} \\
\hline & $\mathrm{c}$ & $\kappa$ & $\mathrm{c}$ & $\kappa$ \\
\hline$E_{1}$ & 0.095 & 0.76 & 0.025 & 0.067 \\
\hline$E_{2}$ & 0.30 & 0.28 & 0.19 & 0.057 \\
\hline$E_{3}$ & 0.42 & 0.24 & 0.29 & 0.051 \\
\hline$E_{4}$ & 0.48 & 0.21 & 0.35 & 0.047 \\
\hline
\end{tabular}

TABLE 2

One can also notice that the nome $q$ is increasing with the symmetry $E_{m}$, i.e., the profile is more soliton-like as $m$ increases. It is because those states with large $m$ are highly excited and classical picture can be well applied. At the hard-wall limit $(a=0)$, the profile $\varepsilon_{\mathrm{p}}(x)$ is given by Eq.(4.5) $\varepsilon_{\mathrm{p}}(x)=\sum_{n=1}^{m}(-1)^{n+1} \frac{\sin 2 n \pi x}{n \pi}$ and $\varepsilon_{\mathrm{p}}(x) \simeq x(|x| \leq 1 / 2)$ as $m \rightarrow \infty$. On the other hand, the fitting function becomes $f_{2}(x ; j=1) \propto x$ as $q \rightarrow 1$. Therefore it is expected that the nome $q \rightarrow 1$ as $m \rightarrow \infty$.

\subsection{Two-soliton state}

So far we have studied $1 \mathrm{~h}$ and $1 \mathrm{p}$ excitations. In order to show the validity of the MPC, we will investigate two-soliton state. In classical mechanics, equally 
spaced two solitons are expressed by the discriminant which has one large nextto-outermost action [22]. In quantum mechanics, this discriminant corresponds to the sequence of quantum numbers $(0,0, \ldots, 0, n, 0)$ with large integer $n$, which has been discussed by Siddharthan and Shastry [14]. In our calculation, one of these sequences is the 3 -rd level of $E_{4}$-symmetry. Although the quantum numbers $(0,0,0,0,0,0,0,0,0,2,0)$ is not so evident to indicate the solitonic discriminant, the MPC of the wave function is expected to have solitonic configuration. Since the energy of two-soliton state is large and the variational wave function is less accurate, we will be contented with this lowest excited two-soliton state.

In the harmonic chain limit $(a \rightarrow \infty)$, the MPC of the wave function can be analytically given in the same way as section $4-2$, i.e.,

$$
y_{i}^{0}=\left(T \boldsymbol{\xi}^{0}\right)_{i}=\sqrt{\frac{2}{N}} \sqrt{\frac{2}{\omega_{2}}} \cos \left(\frac{4 \pi}{N}(i-1)-\alpha\right) .
$$

As is expected, this is the 2-nd harmonics or quadruple deformation [14]. We have calculated the MPC of the 3-rd state of $E_{4}$-symmetry with the range parameter $a$ varied. The MPC $\varepsilon(x)$ is shown in Fig.11. It is clearly seen that $\varepsilon(x)$ is close to $\sin 4 \pi x$ (2-nd harmonics) for large $a$, while $\varepsilon(x)$ tends to cnoidal shape as $a \rightarrow 0$. One can also expect that this cnoidal shape would be more sharpened for higher excited two-soliton states. We have made fittings in terms of elliptic theta function with $1 / 2$ period and obtained fitted nomes as $q=0.037,0.071,0.17,0.28$ for $a=1.5,1.0,0.5,0$ respectively. Thus the profile certainly becomes more peaked form as $a \rightarrow 0$.

If we combine the results of one-soliton and two-soliton states, it is conjectured that classical $j$-soliton state corresponds to the excited state with quantum numbers $(0,0, \ldots, 0, n, 0, \ldots, 0,0)$ where $n$ is a large integer located in the $j$-the column. This state has $E_{j n}$-symmetry and higher excited state with larger $n$ has more soliton-like MPC. 


\section{SUMMARY}

We have studied phonon and soliton type excitations in quantum Toda lattice. The key observation is that the discriminant of the Lax matrix plays essential role both in classical and quantum mechanics. Recently Siddharthan and Shastry [14] have shown, by developing the asymptotic Bethe ansatz method, that there is a close relationship between the classical discriminant and the quantum numbers and 1-hole (particle) type excitations can be identified with the phonon (soliton) -like eigenstates in quantum mechanics. In order to confirm their results in a geometrically more understandable way, we have looked where classical phonon (soliton) -like excitations show up in quantum mechanics.

In order to study geometrical properties, we investigate the most probable configuration (MPC) of eigenstates. The MPC is defined by the configurations which maximize the probability density. In the limiting cases of the Toda lattice, i.e., hard-wall potentials and harmonic chain, the MPC's are calculated analytically and one can estimate the probability around the MPC as well as its extension size. However, for general Toda lattice with a finite potential range, there is no explicit formula of the wave function, and we have performed variational calculations with bases which consist of harmonic chain wave functions.

In a practical example, we have calculated the MPC of the $N=12$ particle Toda lattice, which seems large enough to estimate the properties in the thermodynamic limit. The energy levels are labeled by integer quantum numbers given by Gutzwiller's quantization conditions which are similar to the Bethe ansatz equations. It turns out that the MPC's of 1-hole or 1-particle excitations are certainly well localized and exhaust the probability. This is remarkable since the probability amplitude of an excited state in many-body system usually spreads over whole phase space. We have studied the morphology of the MPC by changing the potential range parameter. Then the profile of the MPC is found to be similar to the configurations of the classical phonon or soliton excitations. Especially the solitonic states, which are buried in the excitation spectra of complicated states, have well-defined configurations similar to the classical soliton of the Toda lattice. We have tried to approximate those MPC's in terms of the elliptic functions which give the cnoidal profile of the classical periodic Toda lattice and obtained reasonable fittings. The fitted parameter nome $q$ becomes close to 1 (extreme anharmonic case) when the nonlinearity of the Toda lattice increases. For phonon excitations, we have found that there are structure changes of the MPC as the potential range increases, i.e., one-node profile goes to $m$-nodes profile $\varepsilon_{\mathrm{h}}(x) \propto \sin 2 \pi m x$ for $E_{m^{-}}$ symmetric state. Another remarkable structure is that $N$ equivalent MPC's, due to the $D_{N}$ symmetry of the Toda lattice, make a ridge in configuration space, and the probability density does not change along this ridge direction.

We have also studied the MPC of $2 \mathrm{~h} 2 \mathrm{p}$ excited state of $E_{4}$-symmetry which corresponds to two-soliton state. Based on the results of one-soliton and twosoliton states, it can be conjectured that the quantum state with only one large quantum number $n$ in the $j$-th column has $j$-solitonic MPC and this MPC tends to more peaked form as $n$ increases. 


\section{APPENDIX A}

\section{Variational calculations}

In this appendix we will describe a way of calculating the matrix elements of the Toda Hamiltonian in terms of harmonic chain bases. Since the symmetric property is determined by the angular parts, the radial parts are not necessarily those of the harmonic chain and, thus, eigenfrequencies should be varied as one of variational parameters.

The kinetic terms are well known since the wave function is the product of harmonic oscillator wave functions. Therefore we will concentrate on the potential terms. Due to the $D_{N}$ symmetry, $N$ potential terms contribute in the same way and we will calculate only the first term $\exp \left(y_{1}-y_{2}\right)$. In terms of normal coordinates they are expressed as

$$
y_{1}-y_{2}=2 \sqrt{\frac{2}{N}} \sum_{k} \sin \frac{k \pi}{N}\left(\sin \frac{k \pi}{N} \xi_{k}^{(1)}-\cos \frac{k \pi}{N} \xi_{k}^{(2)}\right)+\left\{\frac{2}{\sqrt{N}} \xi_{N / 2}\right\} .
$$

Hereafter terms in $\{\cdots\}$ are understood to be present for $N=$ even case. Using the polar coordinate $\left(r_{k}, \theta_{k}\right)$, Eq.(A.1) becomes

$$
y_{1}-y_{2}=2 \sqrt{\frac{2}{N}} \sum_{k} r_{k} \sin \frac{k \pi}{N} \sin \left(\frac{k \pi}{N}-\theta_{k}\right)+\left\{\frac{2}{\sqrt{N}} \xi_{N / 2}\right\} .
$$

Since both the potential term and the basis functions are the product of functions of $\left(r_{k}, \theta_{k}\right)$ and $\left\{\xi_{N / 2}\right\}$ variables, the matrix element is given by the product of each matrix elements. The integral of the angular part is worked out by

$$
\int_{0}^{2 \pi} d \theta \Theta_{\ell_{1}}^{*}(\theta) \exp \left[\alpha \xi \sin \left(\frac{k \pi}{N}-\theta\right)\right] \Theta_{\ell_{2}}(\theta)=\exp \left[i\left(\ell_{2}-\ell_{1}\right)\left(\frac{k \pi}{N}-\frac{\pi}{2}\right)\right] I_{\ell_{2}-\ell_{1}}(\alpha \xi)
$$

with $\alpha=2 \sqrt{\frac{2}{N}} \sin \frac{k \pi}{N}$, and $I_{\ell}(x)$ is the modified Bessel function. The radial part then becomes

$$
\begin{aligned}
& \int_{0}^{\infty} d \xi \xi R_{n_{1} \ell_{1}}(\omega ; \xi) I_{\ell_{2}-\ell_{1}}(\alpha \xi) R_{n_{2} \ell_{2}}(\omega ; \xi) \\
& \quad=N_{n_{1} \ell_{1}} N_{n_{2} \ell_{2}} \omega^{-1} \int_{0}^{\infty} d x x^{\left|\ell_{1}\right|+\left|\ell_{2}\right|+1} e^{-x^{2}} L_{n_{1}}^{\left|\ell_{1}\right|}\left(x^{2}\right) L_{n_{2}}^{\left|\ell_{2}\right|}\left(x^{2}\right) I_{\ell_{2}-\ell_{1}}\left(\frac{\alpha x}{\sqrt{\omega}}\right)
\end{aligned}
$$

One of the ways to calculate the last integral is to make use of the formula

$$
\begin{aligned}
& \int_{0}^{\infty} d x x^{m_{1}+m_{2}+1} e^{-x^{2}} L_{n_{1}}^{m_{1}}\left(x^{2}\right) L_{n_{2}}^{m_{2}}\left(x^{2}\right) I_{m_{1}+m_{2}}(x y) \\
& \left.\quad=\frac{y^{m_{1}+m_{2}}}{2^{m_{1}+m_{2}+1}} \exp \left(\frac{y^{2}}{4}\right) \sum_{K=0}^{n_{1}+n_{2}}(-1)^{K} L_{K}^{m_{1}+m_{2}}\left(-\frac{y^{2}}{4}\right) \sum_{k}\left(\begin{array}{c}
n_{1}+m_{1} \\
n_{1}-k
\end{array}\right)\left(\begin{array}{c}
n_{2}+m_{2} \\
n_{2}-K+k
\end{array}\right)\left(\begin{array}{c}
A_{i}^{K} \\
k
\end{array}\right)\right)
\end{aligned}
$$

with $m_{1}, m_{2}$ non-negative integers. When $\ell_{1}$ and $\ell_{2}$ have the same sign, the integral can be calculated by the recursion formula of the Laguerre polynomial 
starting from $\ell_{1}=0$. However, for large $\left|\ell_{1}\right|$ or $\left|\ell_{2}\right|$ values, it is better to carry out numerical integration since rounding-off errors become large. For $N=$ even case, the matrix element of the $\frac{2}{\sqrt{N}} \xi_{N / 2}$ term is given by

$$
\begin{aligned}
\int_{-\infty}^{\infty} d \xi \varphi_{n_{1}}(\omega ; \xi) \exp \left(\frac{2}{\sqrt{N}} \xi\right) \varphi_{n_{2}}(\omega ; \xi) \\
=\left(\frac{2}{N \omega}\right)^{\left(n_{1}-n_{2}\right) / 2} \frac{\sqrt{n_{2} !}}{\sqrt{n_{1} !}} \exp \left(\frac{1}{N \omega}\right) L_{n_{2}}^{n_{1}-n_{2}}\left(-\frac{2}{N \omega}\right)
\end{aligned}
$$

for $n_{1} \geq n_{2}$ case. 


\section{APPENDIX B}

Most probable configuration for the hard-wall potential

The system of particles interacting with hard-wall potentials is simple enough to express the wave function explicitly, but it still has essential features of many-body system. Therefore it is an important system with its own right and worth studying.

The wave function of the hard-wall potential is the same as that of free fermions or hard-core bosons if the configuration space is restricted to $x_{1}<x_{2}<\cdots<x_{N}$. Thus the wave function is given by the determinant form as ( we set $\hbar=1$ for simplicity)

$$
\Phi=\left(N L^{N}\right)^{-1 / 2}\left|\begin{array}{cccc}
e^{i p_{1} x_{1}} & e^{i p_{2} x_{1}} & \ldots & e^{i p_{N} x_{1}} \\
e^{i p_{1} x_{2}} & e^{i p_{2} x_{2}} & \ldots & e^{i p_{N} x_{2}} \\
\ldots & \ldots & \ldots & \ldots \\
e^{i p_{1} x_{N-1}} & e^{i p_{2} x_{N-1}} & \ldots & e^{i p_{N} x_{N-1}} \\
e^{i p_{1} x_{N}} & e^{i p_{2} x_{N}} & \ldots & e^{i p_{N} x_{N}}
\end{array}\right|
$$

where the momentum $p_{j}$ of the $j$-th particle is $p_{j}=2 \pi n_{j} / L . N$ different integers $\left\{n_{j}\right\}$ are quantum numbers which specify a quantum state. The ground state is $\left\{n_{j}\right\}=(0, \pm 1, \pm 2, \ldots, \pm(N-1) / 2)$ for $N=$ odd, while $\left\{n_{j}\right\}=(0, \pm 1, \pm 2, \ldots, N / 2)$ for $N=$ even. Note that $\Phi$ contains the center-of-mass motion and a common shift of each momentum $p_{j} \rightarrow p_{j}+\Delta p$ does not affect the intrinsic motion. Then the ground state wave function is expressed by the Vandermonde's determinant, apart from a constant factor, as

$$
\Phi_{0}=\left|\begin{array}{ccccc}
1 & z_{1} & z_{1}^{2} & \ldots & z_{1}^{N-1} \\
1 & z_{2} & z_{2}^{2} & \ldots & z_{2}^{N-1} \\
\ldots & \ldots & \ldots & \ldots & \ldots \\
1 & z_{N} & z_{N}^{2} & \ldots & \ldots \\
z_{N}^{N-1}
\end{array}\right|=\prod_{i<j}\left(z_{j}-z_{i}\right)=\Delta_{\mathrm{V}}\left(z_{1}, z_{2}, \ldots, z_{N}\right)
$$

with $z_{j}=e^{i 2 \pi x_{j} / L}$. The probability density $\left|\Phi_{0}\right|^{2}$ further becomes

$$
\left|\Phi_{0}\right|^{2}=\prod_{i<j}\left|z_{j}-z_{i}\right|^{2}=\prod_{i<j} 4 \sin ^{2} \frac{\pi}{L}\left(x_{i}-x_{j}\right)
$$

The energy $E_{0}$ is the sum of kinetic energies of $N$ particles and it becomes $E_{0}=$ $\frac{1}{12} N\left(N^{2}-1\right) \epsilon_{0}$ with $\epsilon_{0}=\frac{1}{2}\left(\frac{2 \pi}{L}\right)^{2}$ in the C.M. system.

The lowest excited state is given by removing the particle on the Fermi energy to the nearest upward state, therefore the quantum numbers are $(0, \pm 1, \ldots, \pm(N-$ $3) / 2,-(N-1) / 2,(N+1) / 2)$ or $(0, \pm 1, \ldots, \pm(N-3) / 2,(N-1) / 2,-(N+1) / 2)$ for $N=$ odd, while $(0, \pm 1, \ldots, \pm(N-2) / 2, N / 2+1)$ or $(0, \pm 1, \ldots, \pm(N-2) / 2,-N / 2-$ 1) for $N=$ even. These two states are degenerate and the excitation energy is $\Delta E=\left(N^{2}-1\right) \epsilon_{0} / N$. The wave function is expressed by the product of the ground state wave function $\Phi_{0}$ and a function $F\left(\left\{x_{i}\right\}\right)$ as

$$
\Phi=F \Phi_{0}, \quad F=\sum_{j} e^{i 2 \pi x_{j} / L}
$$


This factorization comes from the identity

$$
\left|\begin{array}{cccccc}
1 & z_{1} & z_{1}^{2} & \ldots & z_{1}^{N-2} & z_{1}^{N} \\
1 & z_{2} & z_{2}^{2} & \ldots & z_{2}^{N-2} & z_{2}^{N} \\
\ldots & \ldots & \ldots & \ldots & \ldots & \ldots \\
1 & z_{N} & z_{N}^{2} & \ldots & z_{N}^{N-2} & z_{N}^{N}
\end{array}\right|=\left(z_{1}+z_{2}+\cdots+z_{N}\right) \Delta_{\mathrm{V}}\left(z_{1}, z_{2}, \ldots, z_{N}\right)
$$

The probability density is

$$
\begin{aligned}
P\left(x_{1}, x_{2}, \ldots, x_{N}\right) & =\left|\Phi\left(x_{1}, x_{2}, \ldots, x_{N}\right)\right|^{2} \\
& =\left|\Delta_{\mathrm{V}}\left(z_{1}, z_{2}, \ldots, z_{N}\right)\right|^{2} \times \sum_{i, j} 2 \cos \frac{2 \pi}{L}\left(x_{i}-x_{j}\right) \\
& =\prod_{i<j} 4 \sin ^{2} \frac{\pi}{L}\left(x_{i}-x_{j}\right) \times \sum_{i, j} 2 \cos \frac{2 \pi}{L}\left(x_{i}-x_{j}\right) .
\end{aligned}
$$

The MPC $\left\{x_{i}^{0}\right\}$ is a solution which maximizes the probability density $P$, i.e.,

$$
\frac{\partial \ln P}{\partial x_{k}}=\frac{2 \pi}{L} \sum_{j} \cot \frac{\pi}{L}\left(x_{k}-x_{j}\right)-\frac{4 \pi}{L} \frac{\sum_{j} \sin \frac{2 \pi}{L}\left(x_{k}-x_{j}\right)}{\sum_{i, j} \cos \frac{2 \pi}{L}\left(x_{i}-x_{j}\right)}=0 .
$$

We use $\ln P$ instead of $P$ in order to simplify the calculation. It is clear that the equal spacing configuration $\bar{x}_{i}=i / D(D=N / L)$ certainly satisfies the equation corresponding to the ground state $\Phi_{0}$,

$$
\frac{\partial \ln P}{\partial x_{k}}=\frac{2 \pi}{L} \sum_{j} \cot \frac{\pi}{L}\left(x_{k}-x_{j}\right)=0 .
$$

One can suppose that the MPC $\left\{x_{i}^{0}\right\}$ would slightly change from $\bar{x}_{i}=i / D$ since the lowest excited state is different from the ground state only by the prefactor $F$ in Eq.(B.4). Therefore we will determine deviations $\varepsilon_{i} / D=x_{i}^{0}-\bar{x}_{i}$. Our numerical calculations up to $N=20$ indicate $\varepsilon_{i}=O(1)$. By expanding Eq.(B.7) at $\bar{x}_{i}$ and approximating the sum by an integral since we are interested in the thermodynamic limit, i.e., setting $\varepsilon_{i}=\varepsilon(i / N)$ and $\frac{1}{N} \sum_{i} f(i / N)=\int_{-1 / 2}^{1 / 2} f(x) d x$, Eq.(B.7) becomes the integral equation,

$$
-2 \pi^{2} \int_{-1 / 2}^{1 / 2} d x \frac{\varepsilon(y)-\varepsilon(x)}{\sin ^{2} \pi(y-x)}+\frac{2 \sin 2 \pi y}{\int_{-1 / 2}^{1 / 2} d x \varepsilon(x) \sin 2 \pi x}=0,
$$

where we used the properties $\varepsilon(x+1)=\varepsilon(x)$ and $\varepsilon(-x)=-\varepsilon(x)$ due to the $D_{N}$ symmetry. In order to solve the integral equation (B.9), let us expand $\varepsilon(x)(|x| \leq$ $1 / 2$ ) in a Fourier series

$$
\varepsilon(x)=\sum_{n=1}^{\infty} a_{n} \sin 2 n \pi x
$$

Using the principal value integral formula

$$
\mathrm{P} \int_{-1 / 2}^{1 / 2} d x \frac{\sin 2 n \pi y-\sin 2 n \pi x}{\sin ^{2} \pi(y-x)}=2 n \sin 2 n \pi y,
$$


one can obtain the solution

$$
a_{1}=\mp \frac{1}{\pi}, \quad a_{n}=0 \quad(n \geq 2) .
$$

The double sign is $-(+)$ for hole (particle) excitation respectively. Although $1 \mathrm{~h}$ and $1 \mathrm{p}$ excitations are identical for $E_{1}$-symmetry, those signs of Eq.(B.12) are assigned consistently with other $E_{m}$-symmetry $(m \geq 2)$. This simple result is somewhat unexpected.

Next let us examine the Hessian matrix given by

$$
H_{k \ell}=\frac{1}{2}\left(\frac{\partial^{2} \ln P}{\partial x_{k} \partial x_{\ell}}\right)_{\left\{x_{i}^{0}\right\}} .
$$

Once the Hessian matrix is calculated, the probability density near the MPC $\left\{x_{i}^{0}\right\}$ is given by

$$
\begin{aligned}
\left|\Phi\left(\left\{x_{i}\right\}\right)\right|^{2} & =\exp (\ln P) \\
& \simeq \exp \left(\ln P\left(\left\{x_{i}^{0}\right\}\right)+{ }^{t} \overrightarrow{\Delta x} H \overrightarrow{\Delta x}\right) \\
& =\left|\Phi\left(\left\{x_{i}^{0}\right\}\right)\right|^{2} \exp \left({ }^{t} \overrightarrow{\Delta x} H \overrightarrow{\Delta x}\right)
\end{aligned}
$$

with $\Delta x_{k}=x_{k}-x_{k}^{0}$, and $I_{\mathrm{MPC}}$ is approximately estimated by the Gaussian integration

$$
I_{\mathrm{MPC}}=\int\left|\Phi\left(\left\{x_{i}\right\}\right)\right|^{2} d V \simeq\left|\Phi\left(\left\{x_{i}^{0}\right\}\right)\right|^{2} \prod_{k} \sqrt{\frac{\pi}{\left|h_{k}\right|}}
$$

where $\left\{h_{k}\right\}$ are eigenvalues of the Hessian matrix.

In the case of the lowest excited state with $E_{1}$-symmetry, the Hessian matrix turns out, in a relevant order,

$$
H=H_{0}+H_{1}
$$

with

$$
\left(H_{0}\right)_{k \ell}= \begin{cases}-\left(\frac{\pi}{L}\right)^{2} \sum_{j \neq k} \sin ^{-2} \frac{\pi}{N}(k-j) & \text { for } k=\ell \\ \left(\frac{\pi}{L}\right)^{2} \sin ^{-2} \frac{\pi}{N}(k-\ell) & \text { for } k \neq \ell,\end{cases}
$$

and

$$
\left(H_{1}\right)_{k \ell}= \begin{cases}4\left(\frac{\pi}{L}\right)^{2}\left(\cos \frac{2 \pi k}{N}+\cos \frac{4 \pi k}{N}\right) & \text { for } k=\ell \\ 4\left(\frac{\pi}{L}\right)^{2}\left(\cos \frac{2 \pi(k+\ell)}{N}-\frac{2}{N} \sin \frac{2 \pi(k-\ell)}{N}\left(\sin \frac{2 \pi k}{N}-\sin \frac{2 \pi \ell}{N}\right)\right) & \text { for } k \neq \ell .\end{cases}
$$

The matrix $H_{0}$ and $H_{1}$ correspond to the first and second term of Eq.(B.7) respectively. Note that the next order of Eq.(B.17) does not contribute in the large $N$ limit. The eigenvalues $\lambda_{s}$ and eigenvectors $v_{j}^{(s)}$ of the matrix $H_{0}$ are given by 
Calogero-Perelomov's formula [27],

$$
\begin{aligned}
\lambda_{s} & =-2 s(N-s)\left(\frac{\pi}{L}\right)^{2}, \\
v_{j}^{(s)} & =\cos \frac{2 s j \pi}{N}, \quad \sin \frac{2 s j \pi}{N} \quad 0 \leq s \leq[N / 2] .
\end{aligned}
$$

The eigenvalues are generally doubly degenerate except for $s=N / 2(N=$ even $)$ case. The eigenvalue $\lambda_{0}=0(s=0)$ corresponds to the free C.M. motion due to the translational invariance. With eigenvalues $\lambda_{s}=-2 s(N-s)\left(\frac{\pi}{L}\right)^{2}$, one can approximately calculate the normalization of the ground state $\Phi_{0}$ and it turns out by using Stirling's formula

$$
\begin{aligned}
\int\left|\Phi_{0}\left(\left\{x_{i}\right\}\right)\right|^{2} d V & \simeq N^{-1 / 2} L^{-(N-1)}\left|\Phi_{0}\left(\left\{x_{i}^{0}\right\}\right)\right|^{2} \prod_{s=1}^{N-1} \frac{L}{\pi} \sqrt{\frac{\pi}{2 s(N-s)}} \\
& =N^{-1 / 2} N^{N}\left(\frac{1}{\sqrt{2 \pi}}\right)^{N-1} \frac{1}{(N-1) !} \\
& =\left(\frac{e}{\sqrt{2 \pi}}\right)^{N} \simeq(1.08)^{N}
\end{aligned}
$$

where $N^{-1 / 2} L^{-(N-1)}$ is a normalization factor of internal coordinates and

$$
\begin{aligned}
\left|\Phi_{0}\left(\left\{x_{i}^{0}\right\}\right)\right|^{2} & =\prod_{i<j} 4 \sin ^{2} \frac{\pi}{N}(i-j)=2^{N(N-1)}\left[\prod_{j=1}^{N-1} \sin \frac{j \pi}{N}\right]^{N} \\
& =2^{N(N-1)}\left[\frac{N}{2^{N-1}}\right]^{N}=N^{N} .
\end{aligned}
$$

The average $\bar{h}$ of the eigenvalues $\left\{h_{k}\right\}$ of the Hessian matrix, which is defined by the geometrical mean $\bar{h}=\left(\prod_{k}\left|h_{k}\right|\right)^{\frac{1}{N-1}}$, turns out $\bar{h}=\frac{2 \pi^{2} D^{2}}{e^{2}}$ and, thus, the average extension $\bar{r}$ of the Gaussian form is $\bar{r}=1 / \sqrt{\bar{h}}=\frac{e}{\sqrt{2} \pi} \frac{1}{D} \simeq 0.61 / D$. Therefore the extension of the ground state is, roughly speaking, a half of the equilibrium lattice spacing.

When the second term $H_{1}$ is incorporated, one can calculate the eigenvalues of $H=H_{0}+H_{1}$ in the same way as the perturbation method of quantum mechanics. In this case, the total Hessian matrix $H$ still has trivial zero-eigenvalue due to the translational invariance, i.e., $\sum_{\ell} H_{k \ell}=0$ for any $k$. In the large $N$ limit, the perturbative $H_{1}$-term only shifts $s=1$ degenerate eigenvalues of $H_{0}$ as follows,

$$
\begin{aligned}
\lambda_{1} & =-2(N-1)\left(\frac{\pi}{L}\right)^{2} \\
& \rightarrow-2(N-1)\left(\frac{\pi}{L}\right)^{2} \pm 2 N\left(\frac{\pi}{L}\right)^{2}= \begin{cases}2\left(\frac{\pi}{L}\right)^{2} \approx 0 & \text { for } v_{j}^{(1)}=\cos \frac{2 j \pi}{N} \\
-4 N\left(\frac{\pi}{L}\right)^{2} & \text { for } v_{j}^{(1)}=\sin \frac{2 j \pi}{N}\end{cases}
\end{aligned}
$$

and other eigenvalues remain unchanged. It is naturally expected that, apart from the trivial zero-eigenvalue due to the translational invariance, one of the eigenvalues of the Hessian matrix vanishes. There are $N$ equivalent MPC's coming from the $D_{N}$ 
symmetry, which make a continuous ridge in the large $N$ limit, and the direction between the nearest MPC's should be an eigenvector of vanishing eigenvalue of the Hessian matrix. In fact, $\varepsilon_{j}=\frac{1}{\pi} \sin \frac{2 \pi j}{N}$ and the direction of nearest MPC's is in a central difference form

$$
\Delta \varepsilon_{j}=\varepsilon_{j+1}-\varepsilon_{j-1}=\frac{2}{\pi} \sin \frac{2 \pi}{N} \cos \frac{2 \pi j}{N} \propto \cos \frac{2 \pi j}{N},
$$

and thus $\Delta \varepsilon_{j}$ is certainly proportional to the eigenvector $v_{j}^{(1)}$ of the zero-eigenvalue.

One should be careful in calculating $I_{\mathrm{MPC}}$ since one of $\left\{h_{k}\right\}$ vanishes and the Gaussian approximation cannot be applied. We will calculate $I_{\mathrm{MPC}}$ in the intrinsic coordinates in order to avoid trivial zero-eigenvalue due to the C.M. motion. In practice, we use deviations from the equidistance configuration $y_{i}=x_{i}-\bar{x}_{i}$ as intrinsic coordinates $\left(\sum_{i} y_{i}=0\right)$. After diagonalizing the Hessian matrix, the local coordinates $\left\{\xi_{i}\right\}$ around the MPC are given by an orthogonal transformation of $\left\{y_{i}\right\}$. The integrals corresponding to negative eigenvalues are carried out by Gaussian integral. For the zero-eigenvalue flat direction with $\xi_{1}$-coordinate, we can take $\xi_{1}$-coordinate as a global coordinate and will approximate the integral by $N$ times of the linear length between nearest MPC's, i.e.,

$$
\int d \xi_{1}=N \Delta \xi_{1}=N\left(\xi_{1}\left(\left\{y_{i}\right\}\right)-\xi_{1}\left(\left\{y_{i} \rightarrow y_{i+1}\right\}\right)\right) .
$$

Since

$$
\xi_{1}=\sqrt{\frac{2}{N}} \sum_{i} \cos \frac{2 \pi i}{N} \times L y_{i}
$$

then

$$
\begin{aligned}
\Delta \xi_{1} & =\sqrt{\frac{2}{N}} \frac{L}{\pi} \sum_{i} \cos \frac{2 \pi i}{N}\left(\sin \frac{2 \pi(i+1)}{N}-\sin \frac{2 \pi i}{N}\right) \\
& =\sqrt{\frac{2}{N}} \frac{L}{2 \pi} \sin \frac{2 \pi}{N} \approx \sqrt{\frac{2}{N}} \frac{L}{N} \quad(N \rightarrow \infty) .
\end{aligned}
$$

The probability density is calculated to be $\left|\Phi\left(\left\{x_{i}^{0}\right\}\right)\right|^{2}=N^{N} / e$. Thus one can finally obtain

$$
\begin{aligned}
I_{\mathrm{MPC}} & =N^{-1 / 2} L^{-(N-1)} \times N^{N} / e \times\left(\frac{1}{\sqrt{2 \pi}}\right)^{N-2} L^{N-1} \times \frac{1}{(N-2) !} \frac{1}{\sqrt{2 N}} \times \sqrt{\frac{2}{N}} \\
& =\left(\frac{e}{\sqrt{2 \pi}}\right)^{N-1} \simeq(1.08)^{N-1}
\end{aligned}
$$

Since the approximate normalization Eq.(B.21) of the ground state is $\left(\frac{e}{\sqrt{2 \pi}}\right)^{N}$, Eq.(B.28) indicates that the relative ratio is $\left(\frac{e}{\sqrt{2 \pi}}\right)^{-1}=0.92$ and the MPC's exhaust the probability. The average extension of the MPC except for the flat direction is $\bar{r}=\frac{e}{\sqrt{2} \pi} \frac{1}{D}$ in the large $N$ limit, which is the same as the extension of the ground state. 
Now let us evaluate the MPC in general $E_{m}$-symmetry. Before going into the details of the calculation, it should be reminded that the excited states of free fermions can be described by Schur functions [28]. In the case of $E_{1}$-symmetry, lowest 1hole excitation and 1-particle excitation are identical. However, for $E_{m}$-symmetry $(m \geq 2)$ 1-hole excitation and 1-particle excitation are definitely different.

For the $k$-th hole excited state, the wave function is to replace the $k$-th column as $z_{i}^{N-k} \rightarrow z_{i}^{N}$

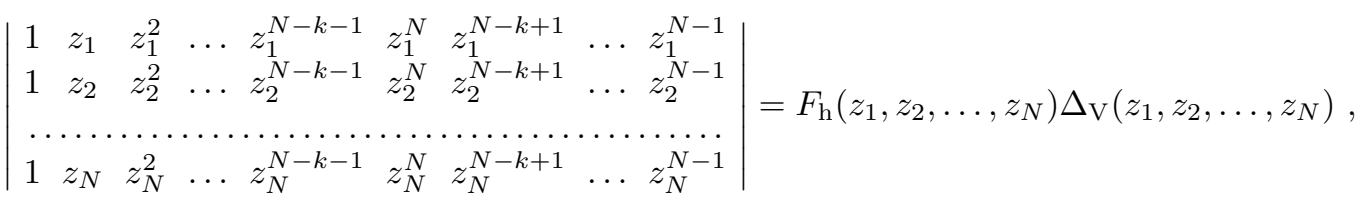

where the function $F_{\mathrm{h}}$ is the $k$-th order symmetric function

$$
F_{\mathrm{h}}\left(z_{1}, z_{2}, \ldots, z_{N}\right)=(-1)^{k+1} \sum_{i_{1}<i_{2}<\cdots<i_{k}} z_{i_{1}} z_{i_{2}} \cdots z_{i_{k}} .
$$

In a similar way, the wave function of $k$-th particle excited state is to replace the last column as $z_{i}^{N-1} \rightarrow z_{i}^{N-1+k}$,

$$
\left|\begin{array}{cccccc}
1 & z_{1} & z_{1}^{2} & \ldots & z_{1}^{N-2} & z_{1}^{N-1+k} \\
1 & z_{2} & z_{2}^{2} & \ldots & z_{2}^{N-2} & z_{2}^{N-1+k} \\
\ldots & \ldots & \ldots & \ldots & \ldots \ldots & \ldots \ldots \\
1 & z_{N} & z_{N}^{2} & \ldots & z_{N}^{N-2} & z_{N}^{N-1+k}
\end{array}\right|=F_{\mathrm{p}}\left(z_{1}, z_{2}, \ldots, z_{N}\right) \Delta_{\mathrm{V}}\left(z_{1}, z_{2}, \ldots, z_{N}\right)
$$

where the function $F_{\mathrm{p}}$ is the $k$-th order symmetric function

$$
F_{\mathrm{p}}\left(z_{1}, z_{2}, \ldots, z_{N}\right)=\sum_{i_{1} \leq i_{2} \leq \cdots \leq i_{k}} z_{i_{1}} z_{i_{2}} \cdots z_{i_{k}} .
$$

The difference of the function $F_{\mathrm{h}}$ and $F_{\mathrm{p}}$ is that there appear only different $z_{k}$ arguments in $F_{\mathrm{h}}$, while there are same $z_{k}$ arguments in $F_{\mathrm{p}}$. For example in the case of $k=3$,

$$
\begin{aligned}
F_{\mathrm{h}}\left(z_{1}, z_{2}, \ldots, z_{N}\right)= & z_{1} z_{2} z_{3}+z_{1} z_{2} z_{4}+\cdots+z_{N-2} z_{N-1} z_{N} \\
F_{\mathrm{p}}\left(z_{1}, z_{2}, \ldots, z_{N}\right)= & z_{1}^{3}+z_{2}^{3}+\cdots+z_{N}^{3}+z_{1}^{2} z_{2}+\cdots+z_{N-1} z_{N}^{2} \\
& +z_{1} z_{2} z_{3}+\cdots+z_{N-2} z_{N-1} z_{N}
\end{aligned}
$$

These two formulas (B.29-B.32) are simple examples of Schur functions.

In order to calculate the MPC $\left\{x_{i}^{0}\right\}$, it is preferable to employ the functional derivative method. In the case of $E_{1}$-symmetry, the first term of Eq.(B.9) comes from the ground state wave function and is always present also in $E_{m}$-symmetric case, while the second term corresponds to the prefactor $F$. Since

$$
\sum_{j} e^{2 \pi i x_{j} / L}=\sum_{j} e^{2 \pi i\left(j+\varepsilon_{j}\right) / N}=N \int d x e^{2 \pi i x} e^{2 \pi i \varepsilon(x) / N}
$$


and

$$
\left|\sum_{j} e^{2 \pi i x_{j} / L}\right|^{2}=N^{2} \int d x d y e^{2 \pi i(x-y)} e^{2 \pi i(\varepsilon(x)-\varepsilon(y)) / N},
$$

then the derivative with respect to $x_{k}$ is replaced by the functional derivative, i.e., $\frac{\partial}{\partial x_{k}} \rightarrow \frac{\delta}{L \delta \varepsilon(y)}$ and

$\frac{\delta}{\delta \varepsilon(y)} \ln \left[N^{2} \int d x d y e^{2 \pi i(x-y)} e^{2 \pi i(\varepsilon(x)-\varepsilon(y)) / N}\right]=\frac{2 \sin 2 \pi y}{\int_{-1 / 2}^{1 / 2} d x \varepsilon(x) \sin 2 \pi x}+O(1 / N)$,

which is the same result as Eq.(B.9). For the calculation of the Hessian matrix, the functional derivative method certainly gives the same results as Eq.(B.18).

In the case of $E_{m}$-symmetry $(m=2,3,4)$, the prefactor $F\left(\left\{x_{i}\right\}\right)$ is calculated in the same way as $E_{1}$-symmetry, although it becomes more and more involved. Let us write down final formulas.

$$
\begin{aligned}
& E_{2}: F=\frac{1}{2}\left(I_{1}^{2} \mp I_{2}\right), \\
& E_{3}: F=\frac{1}{6}\left(I_{1}^{3} \mp 3 I_{1} I_{2}+2 I_{3}\right), \\
& E_{4}: F=\frac{1}{24}\left(I_{1}^{4} \mp 6 I_{4}+8 I_{1} I_{3}+3 I_{2}^{2} \mp 6 I_{1}^{2} I_{2}\right),
\end{aligned}
$$

where double sign is $-(+)$ for $1 \mathrm{~h}(1 \mathrm{p})$ excitation respectively, and $I_{n}$ is defined by

$$
I_{n}=\sum_{j} z_{j}^{n}=\sum_{j} e^{2 n \pi i x_{j} / L}=N \int d x e^{2 n \pi i x} e^{2 n \pi i \varepsilon(x) / N} .
$$

One can notice the similarity between $1 \mathrm{~h}$ and $1 \mathrm{p}$ excitations which just differ in some relative signs. The calculation of the functional derivative $\frac{\delta}{\delta \varepsilon(y)}$ is involved but straightforward.

The solutions of the integral equation are given by the Fourier expansion in the similar way as $E_{1}$-symmetric case. The results turn out to be surprisingly simple. For $1 \mathrm{~h}$ excitations,

$$
\begin{aligned}
& E_{2}: \varepsilon_{\mathrm{h}}(x)=-\frac{1}{\pi} \sin 2 \pi x+\frac{1}{2 \pi} \sin 4 \pi x \\
& E_{3}: \varepsilon_{\mathrm{h}}(x)=-\frac{1}{\pi} \sin 2 \pi x+\frac{1}{2 \pi} \sin 4 \pi x-\frac{1}{3 \pi} \sin 6 \pi x \\
& E_{4}: \varepsilon_{\mathrm{h}}(x)=-\frac{1}{\pi} \sin 2 \pi x+\frac{1}{2 \pi} \sin 4 \pi x-\frac{1}{3 \pi} \sin 6 \pi x+\frac{1}{4 \pi} \sin 8 \pi x,
\end{aligned}
$$

and, for $1 \mathrm{p}$ excitations, $\varepsilon_{\mathrm{p}}(x)$ is given by $\varepsilon_{\mathrm{p}}(x)=-\varepsilon_{\mathrm{h}}(x)$. Up to $E_{4}$-symmetry, we have explicitly solved the integral equation by the Fourier expansion. As it is clear from the above formulas, one can conjecture that the solution for $E_{m}$-symmetry is

$$
\varepsilon_{\mathrm{h}}(x)=\sum_{n=1}^{m}(-1)^{n} \frac{1}{n \pi} \sin 2 n \pi x, \quad \varepsilon_{\mathrm{p}}(x)=-\varepsilon_{\mathrm{h}}(x), \quad(|x| \leq 1 / 2) .
$$


As for the Hessian matrix, the calculation of functional derivative is rather involved. At the end, non-vanishing order- $N$ matrix elements of $H_{1}$ in Eq.(B.16) corresponding to the prefactor $F\left(\left\{x_{i}\right\}\right)$ are only first $m \times m$ block in the large $N$ limit for the case of $E_{m}$-symmetry. For example, for $m=2$,

$$
H_{1}=\left(\frac{\pi}{L}\right)^{2}\left(\begin{array}{cccc}
0 & -2 N & 0 & \ldots \\
-2 N & 2 N & 0 & \ldots \\
0 & 0 & 0 & \ldots \\
\ldots & \ldots & \ldots & \ldots
\end{array}\right) \text { for } \quad v_{j}^{(s)}=\cos \frac{2 s j \pi}{N}
$$

and

$$
H_{1}=\left(\frac{\pi}{L}\right)^{2}\left(\begin{array}{cccc}
-2 N & 2 N & 0 & \ldots \\
2 N & -6 N & 0 & \ldots \\
0 & 0 & 0 & \ldots \\
\ldots & \ldots & \ldots & \ldots
\end{array}\right) \quad \text { for } \quad v_{j}^{(s)}=\sin \frac{2 s j \pi}{N}
$$

By diagonalizing the Hessian matrix $H=H_{0}+H_{1}$, one can obtain eigenvalues and eigenvectors. It turns out from the calculation up to $m=4$ that one of the eigenvalues always vanishes in the large $N$ limit and the eigenvector of the zeroeigenvalue is the direction of the nearest neighbour MPC's. These situations are the same as those of $E_{1}$-symmetry and one can expect that it is a common feature for general $E_{m}$-symmetric state.

The calculation of $I_{\mathrm{MPC}}$ is also carried out in the same way as $E_{1}$-symmetry. The result is

$$
I_{\mathrm{MPC}}=\left(\frac{e}{\sqrt{2 \pi}}\right)^{N-1} \times \frac{m}{\exp \left(\frac{1}{2}+\frac{1}{3}+\cdots+\frac{1}{m}\right)} \quad(m \geq 2)
$$

and $I_{\mathrm{MPC}} \approx\left(\frac{e}{\sqrt{2 \pi}}\right)^{N-1} e^{1-\gamma_{\mathrm{E}}}$ for large $m . \quad\left(\gamma_{\mathrm{E}}=0.5772 \ldots\right.$ is Euler's constant. $)$ Since the approximate normalization of the ground state is $\left(\frac{e}{\sqrt{2 \pi}}\right)^{N}$, the relative ratio is $\left(\frac{e}{\sqrt{2 \pi}}\right)^{-1} e^{1-\gamma_{\mathrm{E}}} \simeq 1.41$. Therefore one can conclude that the MPC's certainly fill the probability for general $E_{m}$-symmetric state. 


\section{APPENDIX C}

Solution of the classical periodic Toda lattice with genus $g=1$

In this appendix, we will review the explicit solution of the genus $g=1$ classical periodic Toda lattice. Although general initial value problems have been solved in terms of the generalized Riemann's theta function [6], it is instructive to construct the $g=1$ solution explicitly in terms of the elliptic function. The derivation of the equation of motion of the periodic Toda lattice is rather involved and we will skip the way of canonical transformation $\left(p_{i}, q_{i}\right) \rightarrow\left(\nu_{i}, \mu_{i}\right)$, which is described in Ref.[1, 23] for example.

The equation of motion of the auxiliary spectra $\left\{\mu_{i}\right\}$ turns out

$$
\frac{d \mu_{k}}{d t}= \pm \frac{2 \sqrt{R\left(\mu_{k}\right)}}{\prod_{\ell \neq k}\left(\mu_{k}-\mu_{\ell}\right)},
$$

where $R(\lambda)=\prod_{j=1}^{2 g+2}\left(\lambda-\lambda_{j}\right)$ and $\left\{\lambda_{j}\right\}$ are non-degenerate roots of $\Delta(\lambda)= \pm 2$. In the case of $g=1$, there is only one dynamical degree of freedom expressed by an auxiliary spectrum $\mu$ and other auxiliary spectra are left fixed to the degenerate $\lambda_{j}$ values. The denominator of RHS is $\prod_{\ell \neq k}\left(\mu_{k}-\mu_{\ell}\right)=1$ and the equation is integrated as

$$
\int \frac{d \mu}{\sqrt{R(\mu)}}= \pm 2 t+\text { const. }
$$

For $g=1$ there are 4 non-degenerate roots $\lambda_{j}\left(\lambda_{1}<\lambda_{2}<\lambda_{3}<\lambda_{4}\right)$ and the auxiliary spectrum $\mu$ moves in the interval $\lambda_{2} \leq \mu \leq \lambda_{3}$. Since $R(\mu)=\left(\mu-\lambda_{1}\right)(\mu-$ $\left.\lambda_{2}\right)\left(\mu-\lambda_{3}\right)\left(\mu-\lambda_{4}\right)$, the integral of LHS can be expressed in terms of the elliptic function by using the formula

$$
\begin{aligned}
& \int_{\lambda_{2}}^{\mu} \frac{d x}{\sqrt{\left(x-\lambda_{1}\right)\left(x-\lambda_{2}\right)\left(x-\lambda_{3}\right)\left(x-\lambda_{4}\right)}} \\
& \quad=\frac{2}{\sqrt{\left(\lambda_{4}-\lambda_{2}\right)\left(\lambda_{3}-\lambda_{1}\right)}} \operatorname{sn}^{-1}\left(\sqrt{\frac{\left(\lambda_{3}-\lambda_{1}\right)\left(\mu-\lambda_{2}\right)}{\left(\lambda_{3}-\lambda_{2}\right)\left(\mu-\lambda_{1}\right)}}, k\right),
\end{aligned}
$$

where modulus is $k=\left(\frac{\left(\lambda_{3}-\lambda_{2}\right)\left(\lambda_{4}-\lambda_{1}\right)}{\left(\lambda_{4}-\lambda_{2}\right)\left(\lambda_{3}-\lambda_{1}\right)}\right)^{1 / 2}$. The large gap $\left[\lambda_{2}, \lambda_{3}\right]$ corresponds to the large modulus $k$. The period $T$ is

$$
T=\int_{\lambda_{2}}^{\lambda_{3}} \frac{d \mu}{\sqrt{R(\mu)}}=\frac{4 K(k)}{\sqrt{\left(\lambda_{4}-\lambda_{2}\right)\left(\lambda_{3}-\lambda_{1}\right)}},
$$

where $K(k)$ is a complete integral of the first kind for the modulus $k$. Then $\mu(t)$ is expressed as

$$
\begin{aligned}
\mu(t) & =\lambda_{1}+\frac{\left(\lambda_{1}-\lambda_{2}\right)\left(\lambda_{3}-\lambda_{1}\right)}{\left(\lambda_{3}-\lambda_{2}\right) \operatorname{sn}^{2}(u, k)-\left(\lambda_{3}-\lambda_{1}\right)} \\
& =\lambda_{2}+\sqrt{\left(\lambda_{4}-\lambda_{2}\right)\left(\lambda_{3}-\lambda_{1}\right)}\left(Z(a)-\frac{1}{2} Z(u+a)+\frac{1}{2} Z(u-a)\right)
\end{aligned}
$$


where $u=\sqrt{\left(\lambda_{4}-\lambda_{2}\right)\left(\lambda_{3}-\lambda_{1}\right)} t$ and $a$ is defined by $\operatorname{sn}^{2} a=\frac{\left(\lambda_{4}-\lambda_{2}\right)}{\left(\lambda_{4}-\lambda_{1}\right)} . Z(x)$ is Jacobi's zeta function. The auxiliary variable $\mu$ is related to the original variables $\left(p_{i}, q_{i}\right)$ as

$$
\frac{1}{2} p_{1}=\frac{1}{2} \dot{q}_{1}=\frac{1}{2} \sum_{k=1}^{4} \lambda_{k}-\mu
$$

The time-independent term of Eq.(C.6) should identically vanish in the C.M. system, i.e.,

$$
\frac{1}{2} \sum_{k=1}^{4} \lambda_{k}-\lambda_{2}-\sqrt{\left(\lambda_{4}-\lambda_{2}\right)\left(\lambda_{3}-\lambda_{1}\right)} Z(a)=0
$$

due to the periodicity of the solution. We could not prove Eq.(C.7) directly, although they are checked numerically in various initial conditions. Using the relation $Z(x)=\frac{d}{d x} \ln \theta_{0}\left(\frac{x}{2 K}\right)$, where the elliptic theta function $\theta_{0}(u)$ is defined by $\theta_{0}(u)=\sum_{n=-\infty}^{\infty}(-1)^{n} q^{n^{2}} e^{2 n u \pi i}$, one finally obtains

$$
\begin{aligned}
& p_{1}=\sqrt{\left(\lambda_{4}-\lambda_{2}\right)\left(\lambda_{3}-\lambda_{1}\right)}(Z(u+a)-Z(u-a)) \\
& q_{1}=\ln \left\{\theta_{0}\left(\frac{u+a}{2 K}\right) / \theta_{0}\left(\frac{u-a}{2 K}\right)\right\} .
\end{aligned}
$$

When the gap $\left[\lambda_{2}, \lambda_{3}\right]$ is situated in outermost, there is a relation

$$
N a=K
$$

which guarantees $\sum_{k} q_{k}=0$. For $N=3$, Eq.(C.10) can be proved directly, and we have checked numerically its validity up to $N=6$. The position of the $k$-th particle is given by appropriately shifting the phase of the argument, i.e.,

$$
q_{k}=\ln \left\{\theta_{0}\left(\frac{u+a}{2 K}-\frac{k-1}{N}\right) / \theta_{0}\left(\frac{u-a}{2 K}-\frac{k-1}{N}\right)\right\} .
$$

If the gap is located in $j$-th interval from the outermost, then Eq.(C.11) should be modified to

$$
q_{k}=\ln \left\{\theta_{0}\left(\frac{u+a}{2 K}-j \frac{k-1}{N}\right) / \theta_{0}\left(\frac{u-a}{2 K}-j \frac{k-1}{N}\right)\right\},
$$

and there is a relation

$$
N a=j K
$$

The $j$-th inner gap excitation has $j$ nodes in a half period and corresponds to the $j$-th normal mode of the vibration.

The dispersion relation between $\alpha=2 K \nu$ and $\lambda=N / j$ is

$$
2 K \nu=\theta_{0}\left(\frac{1}{\lambda}\right) \operatorname{sn}\left(\frac{2 K}{\lambda}\right) / \theta_{0}(0) .
$$


The limits of large or small modulus are

$$
2 K \nu=\left\{\begin{aligned}
\sin \left(\frac{2 K}{\lambda}\right), & k \rightarrow 0 \\
\sinh \left(\frac{2 K}{\lambda}\right), & k \rightarrow 1
\end{aligned}\right.
$$

Then one can recognize that the cnoidal wave looks like 1-hole excitation at small $k$, while it is like 1-particle excitation at large $k$. 


\section{REFERENCES}

1. M. Toda, J.Phys.Soc.Japan 22 (1967), 431 ;

"Theory of nonlinear lattices", Springer, Berlin, 1981.

2. M. Hénon, Phys.Rev. B 9 (1974), 1921.

3. H. Flaschka, Phys.Rev. B 9 (1974), 1924.

4. H. Flaschka, Prog.Theor.Phys. 51 (1974), 703.

5. M. Kac and P. van Moerbeke, Proc.Nat.Acad.Sci. U.S.A. 72 (1975), 1627, 2879.

6. E. Date and S. Tanaka, Prog.Theor.Phys. 55 (1976), 457;

Prog.Theor.Phys.Suppl. 59 (1976), 107.

7. M.C. Gutzwiller, Ann.Phys. (NY) 124 (1980), 347 ; 133 (1981), 304.

8. A. Matsuyama, Phys.Lett. A 161 (1991), 124

9. A. Matsuyama, Ann.Phys. (NY) 220 (1992), 300

10. E.K. Sklyanin, in "Lecture Notes in Physics", Vol.226, Springer, Berlin, 1985.

11. B. Sutherland, Rocky Mount.J.Math. 8 (1978), 413

12. F.G. Mertens, Z.Phys. B 55 (1984), 353

F.G. Mertens and M. Hader, Z.Phys. B 69 (1984), 121

M. Hader and F.G. Mertens, J.Phys. A 19 (1986), 1913

13. A. Matsuyama, J.Phys. A 29 (1996), 4837

14. R. Siddharthan and B.S. Shastry, Phys.Rev. B 55 (1997), 12196

15. J. Schliemann and F.G. Mertens, J.Phys. A 32 (1999), 5823

16. D.A. Kessler and H. Levine, Phys.Lett. A 81 (1981), 9

I. Andrić, Phys.Lett. A 93 (1983), 275

17. A.P. Polychronakos, Phys.Rev.Lett. 74 (1995), 5153

I. Andrić, V. Bardek and L. Jonke, Phys.Lett. B 357 (1995), 374

18. A. Jevicki and B. Sakita, Nucl.Phys. B 165 (1980), 511

19. B. Sutherland and J. Campbell, Phys.Rev. B 50 (1994), 888

20. F.A. Smirnov, J.Phys. A 31 (1998), 8953

21. S. Kharchev and D. Lebedev, Lett.Math.Phys. 50 (1999), 53

22. W.E. Ferguson, H. Flaschka and D.W. McLaughlin, J.Comput.Phys. 45 (1982), 157

23. H. Flaschka and D.W. McLaughlin, Prog.Theor.Phys. 55 (1976), 438

24. V. Pasquier and M. Gaudin, J.Phys. A 25 (1992), 5243

25. A. Matsuyama, J.Phys. A 29 (1996), 1089

26. M. Fowler and H. Frahm, Phys.Rev. B 39 (1989), 11800

27. I.S. Gradshteyn and I.M. Ryzhik,

"Tables of integrals, series, and products", Academic Press, Inc.

28. I.G. Macdonald, "Symmetric Functions and Hall Polynomials", Oxford Univ. Press, New York, 1995 
Table Caption:

Table1:

Quantum numbers of low energy levels for each symmetry. One of a pair of degenerate quantum numbers is listed. For $E_{4}$-symmetry, the upper (lower) quantum numbers of 5 -th level corresponds to the range $a \leq 0.55(a \geq 0.55)$ respectively.

\begin{tabular}{ccccc}
\hline Level & $E_{1}$ & $E_{2}$ & $E_{3}$ & $E_{4}$ \\
\hline 1 & $(0,0,0,0,0,0,0,0,0,0,1)$ & $(0,0,0,0,0,0,0,0,0,1,0)$ & $(0,0,0,0,0,0,0,0,1,0,0)$ & $(0,0,0,0,0,0,0,1,0,0,0)$ \\
2 & $(1,0,0,0,0,0,0,0,0,1,0)$ & $(0,0,0,0,0,0,0,0,0,0,2)$ & $(0,0,0,0,0,0,0,0,0,1,1)$ & $(0,0,0,0,0,0,0,0,1,0,1)$ \\
3 & $(1,0,0,0,0,0,0,0,0,0,2)$ & $(1,0,0,0,0,0,0,0,1,0,0)$ & $(0,0,0,0,0,0,0,0,0,0,3)$ & $(0,0,0,0,0,0,0,0,0,2,0)$ \\
4 & $(0,1,0,0,0,0,0,0,1,0,0)$ & $(1,0,0,0,0,0,0,0,0,1,1)$ & $(1,0,0,0,0,0,0,1,0,0,0)$ & $(0,0,0,0,0,0,0,0,0,1,2)$ \\
5 & $(2,0,0,0,0,0,0,0,1,0,0)$ & $(1,0,0,0,0,0,0,0,0,0,3)$ & $(1,0,0,0,0,0,0,0,1,0,1)$ & $(1,0,0,0,0,0,1,0,0,0,0)$ \\
& & & & $(0,0,0,0,0,0,0,0,0,0,4)$ \\
\hline
\end{tabular}

Table2:

Parameters of fitting nome $q$ as $q(a)=c e^{-\kappa a}$

\begin{tabular}{ccccc}
\hline & \multicolumn{2}{c}{$0 \leq a \leq 1$} & \multicolumn{2}{c}{$5 \leq a \leq 10$} \\
\hline$E_{m}$ & $\mathrm{c}$ & $\kappa$ & $\mathrm{c}$ & $\kappa$ \\
\hline$E_{1}$ & 0.095 & 0.76 & 0.025 & 0.067 \\
$E_{2}$ & 0.30 & 0.28 & 0.19 & 0.057 \\
$E_{3}$ & 0.42 & 0.24 & 0.29 & 0.051 \\
$E_{4}$ & 0.48 & 0.21 & 0.35 & 0.047 \\
\hline
\end{tabular}




\section{Figure Captions}

Fig.1

Schematic illustrations of discriminants $\Delta(\lambda)$ for phonon/soliton excitations for $N=12$ particle Toda lattice.

Fig.2

Relationship between classical discriminant (upper), semiclassical discriminant (middle) and rapidity distribution (lower) for ground state, hole-excited state and particleexcited state.

Fig.3

Rapidity distributions of typical excitations. $n$-th site corresponds to the Fermi energy.

Fig.4

Low energy levels for each symmetry.

Fig.5

Probabilities of harmonic chain bases for low energy eigenfunctions of $E_{4}$-symmetry. 5 th/6th-level corresponds to the $1 \mathrm{p}$ excitation. Quantum numbers of harmonic chain and explanations of marks are given in lower-right part.

Fig.6

Convergence of the MPC profile of 1-hole excitation in the case of $E_{1}$ and $E_{2^{-}}$ symmetry for the potential range $a=0.5$. The number of particles is indicated in graphs.

Fig.7

Most probable configurations of 1-hole (Fig.7a) and 1-particle (Fig.7b) excitations. Numbers in graphs indicate the potential range $a$. Particle sequences are conveniently relabeled as $i \rightarrow i+6(\bmod 12)$ between Fig.7a and Fig.7b.

Fig.8

Dependences of most probable configurations of 1-hole (Fig.8a) and 1-particle (Fig.8b) excitations on the potential range $a$. Numbers in graphs indicate particle sequence $i$.

Fig.9

Probability density distributions of $E_{1}$-symmetric state of $N=3$ Toda lattice plotted for range parameters $a=0.5,1,5$ and 10. Intrinsic coordinates are $\xi_{1}=$ $\frac{1}{\sqrt{6}}\left(2 x_{1}-x_{2}-x_{3}\right)$ and $\xi_{2}=\frac{1}{\sqrt{2}}\left(x_{2}-x_{3}\right)$. 
Fig.10

Fittings of most probable configurations of $1 p$ state in terms of elliptic theta function. The potential range is $a=0.5$.

Fig.11

Most probable configuration of the 3-rd level of $E_{4}$-symmetry. Numbers in graphs indicate the potential range $a$ and smooth curves are interpolations given by the Spline function. 